\title{
ALFABETIZACIÓN EN EUSKARA EN LAS ESCUELAS RURALES DEL PAÍS VASCO (1900-1939)*
}

\author{
Basque-language literacy in rural schools in the Basque Country \\ (1900-1939)
}

\section{Joxe Garmendia Larrañaga ${ }^{\&}$; Iñaki Zabaleta $\operatorname{Imaz}^{\mathfrak{f}}$ e Hilario Murua Cartón ${ }^{¥}$}

Fecha de recepción: 06/03/2017 • Fecha de aceptación: 02/10/2017

Resumen. En el primer tercio del siglo xx se produce en el País Vasco un proceso de modernización que afectó a la enseñanza en general. En esta época en las zonas rurales todavía había altas tasas de analfabetismo y la población era vascoparlante. Con el objetivo de paliar esta situación las diputaciones de Bizkaia y Gipuzkoa pusieron en marcha dos experiencias: Las Escuelas de Barriada en Bizkaia (1919-1938) y las Escuelas Rurales Provinciales en Gipuzkoa (1930-1938). Aunque en sus inicios, las escuelas de barriada de Bizkaia pretendieron desarrollar una escuela vasca y un proyecto bilingüe, los cambios políticos condicionaron este proyecto. En el caso de las escuelas provinciales de Gipuzkoa, el objetivo era suplir la insuficiencia de instrucción. El objetivo de este trabajo es comparar estas dos experiencias de alfabetización desarrolladas en un ámbito rural, en las que se interrelacionan la alfabetización en castellano y en euskara.

Palabras clave: Escuela rural; País Vasco; Alfabetización; Identidad nacional.

\footnotetext{
* Este artículo es resultado de un proyecto de investigación financiado por el Ministerio de Economía y Competitividad, proyecto número EDU2013-44129-P. Los autores son miembros del Grupo de Estudios Históricos y Comparados en Educación - Garaian, reconocido por el Gobierno Vasco con el número IT 911-16.La versión en euskera de este artículo se publicará en el n. ${ }^{\circ} 8$ de la revista.

\& Departamento de Teoría e Historia de la Educación. Universidad del País Vasco/Euskal Herriko Unibertsitatea. Avda. de Tolosa, 70, 20018 Donostia-San Sebastián. España. joxe.garmendia@ehu.eus

£ Departamento de Teoría e Historia de la Educación Universidad del País Vasco/Euskal Herriko Unibertsitatea. Avda. de Tolosa, 70. 20018 Donostia-San Sebastián. España. i.zabaleta@ehu.eus

${ }^{*}$ Departamento de Teoría e Historia de la Educación. Universidad del País Vasco/Euskal Herriko Unibertsitatea. Avda. de Tolosa, 70. 20018 Donostia-San Sebastián, España. hilario.murua@ehu.eus
}

Cómo citar este artículo: Garmendia Larrañaga, Joxe, Iñaki Zabaleta Imaz e Hilario Murua Cartón. "Alfabetización en Euskara en las escuelas rurales del País Vasco (1900-1939)». Historia y Memoria de la Educación 7 (2018): 191-233. 
Abstract. In the first third of the twentieth century, the Basque Country underwent a modernizing process that affected general education. Given that there were still high rates of illiteracy in rural areas and the populations were Basque-speaking, in an attempt to increase literacy the provincial councils of Bizkaia and Gipuzkoa put two different programmes into motion. Bizkaia established "escuelas de barriada», or neighbourhood schools (1919-1938), and Gipuzkoa instituted the rural provincial schools (1930-1938). Although the neighbourhood schools in Bizkaia began by trying to create Basque schools that were bilingual, political changes influenced the project. In the case of the provincial schools in Gipuzkoa, the goal was to combat the lack of instruction. The objective of this study is to compare these two rural literacy programmes in which literacy in Spanish and in Basque were intertwined.

Keywords: Rural schools; Basque Country; Literacy; National identity.

\section{INTRODUCCIÓN}

A principios del siglo Xx el mundo rural del País Vasco, a pesar del proceso de industrialización, era fiel reflejo de una sociedad tradicional basada en la agricultura y en el que primaba la pobreza. La vida social estaba fundamentada en la familia, el catolicismo, las costumbres y una cultura tradicional propia del mundo rural, donde el euskera era la lengua habitual. La ubicación geográfica de la mayoría de los caseríos vascos, que se encontraban en zonas muy alejadas de los núcleos urbanos, favorecía el mantenimiento de los valores religiosos tradicionales y las costumbres rurales. En este contexto se fue produciendo, gradual y pausadamente, un profundo cambio socio económico. Poco a poco, el mundo rural fue evolucionando en sus estructuras, convirtiéndolo cada vez más en una sociedad urbana e industrial pero manteniendo, en parte, los modos de vida y una mentalidad rural. ${ }^{1}$ Buena muestra de ello fueron la presencia del caciquismo y el monopolio del poder político por parte las fuerzas políticas tradicionales. En el medio rural era patente la influencia política de los grandes propietarios y del clero, que controlaban el poder local y provincial. ${ }^{2}$

\footnotetext{
${ }^{1}$ Jesús Arpal, Begoña Asua y Paulí Davila, Sociedad y Educación en el País Vasco (San Sebastián: Txertoa, 1982).

2 Joxe Garmendia, La enseñanza rural en Gipuzkoa. La labor de la Diputación y los Ayuntamientos, 1900-1950 (Bilbao: Universidad del País Vasco, 2004), 63. El clero seguía siendo pieza clave de casi todas las decisiones. Para profundizar en este tema se puede consultar Luis Castells, Modernización
} 
La profunda transformación socio-económica que se estaba dando en el País Vasco afectó también, y directamente, al modelo educativo con una la baja tasa de alfabetización y escolarización, aunque superior al resto del Estado español. Teniendo en cuenta que la escuela tiene como objetivo primordial estos procesos de alfabetización y escolarización, en el caso vasco se daba, además, la particularidad de que la enseñanza se impartía en una lengua que no era la habitual de la mayoría de la población rural de Bizkaia y Gipuzkoa. Para paliar esta situación, desde las perspectivas fueristas y nacionalistas, se plantearon diversas propuestas. Se trataba de alternativas que potenciaron experiencias paralelas de alfabetización rural a través de estas escuelas, cuyo desarrollo estuvo sujeto siempre a las vicisitudes políticas.

La primera experiencia promovida en el ámbito rural por las instituciones provinciales fueron las escuelas de barriada de Bizkaia, después vendrían las escuelas rurales de Gipuzkoa. En estas escuelas, por lo que respecta a los planteamientos educativos, se tuvieron muy en cuenta las aportaciones pedagógicas de la época y las realizadas en los diversos congresos promovidos por la Sociedad de Estudios Vascos. En estos congresos se planteó la necesidad de desarrollar una política educativa acorde a la realidad del país y para ello se propuso impulsar la lengua vasca en las escuelas rurales. Los promotores y dinamizadores de estos planteamientos fueron, fundamentalmente, organizaciones nacionalistas vascas. Esas fuerzas reivindicaban la enseñanza impartida en euskera, como un elemento básico para el reforzamiento de la cultura y de la identidad vasca. Pero en esta labor se vieron obstaculizadas, entre otros, por las fuerzas monárquicas, que veían en la misma una forma de extender el ideario nacionalista. En consecuencia, los planteamientos educativos de estas escuelas estarán sujetos a los cambios políticos, condicionando su implantación y expansión. Comparar ambas experiencias es el objetivo fundamental de esta aportación.

\section{LA ALFABETIZACIÓN EN EL ÁMBITO RURAL}

El siglo XIX marca el inicio de la estatalización de la enseñanza dentro del proceso de construcción de los sistemas educativos nacionales. Se

y dinámica política en la sociedad Guipuzcoana de la Restauración (1876-1915) (Madrid: Siglo XXI, 1987). 
trataba de materializar la idea de nación, entendida como sujeto político y la construcción de un sistema educativo capaz de formar la ciudadanía en el territorio determinado. Hobsbawm se referirá, como es conocido, al concepto de "fabricar naciones» y situará el trabajo que desempeñará la escuela dentro de la ingeniería política que los Estados pusieron en marcha con ese fin. ${ }^{3}$

En los casos de España y Francia se defendió la lengua nacional y los sistemas educativos comunes en el marco de las fronteras estatales perfectamente delimitadas; al mismo tiempo se intentó - y se logró- crear sistemas legales comunes. ${ }^{4}$ Se ha argumentado que esas medidas eran demandadas por una necesidad urgente de homogeneización cultural, medidas que cobran sentido en el contexto del proceso de modernización que se irá desarrollando y que exigirá individuos alfabetizados en la lengua del Estado para compartir una cultura homogénea transmitida a través de la escuela. La visión de Gellner, pone en evidencia, en cualquier caso, la importancia de la cultura y el idioma en relación al proceso de nacionalización llevado por los Estados a través del sistema educativo. ${ }^{5}$

En este contexto, tanto el Estado francés como el español tuvieron que hacer frente a un reto: la necesidad de materializar, de hacer real, la noción de comunidad y, con tal propósito, tanto la cultura como los idiomas francés y español cobraron una importancia fundamental. En definitiva, las políticas educativas desplegadas por ambos Estados, en esa perspectiva, han estado orientadas a la unidad de los ciudadanos que compondrían el estado-nación y, en ese sentido, la unidad de cultura e idioma ha primado en la política de cada uno de ellos. En el caso del Estado español, hay que señalar la dificultad que existía para la construcción de la identidad nacional; ya que la ideología nacionalista española estaba escasamente vinculada con los proyectos democráticos y modernizadores. ${ }^{6}$ En este contexto hay que destacar que, aunque la enseñanza en las escuelas del País vasco se haya impartido mayoritariamente en

\footnotetext{
${ }^{3}$ Eric J. Hobsbawm, Naciones y nacionalismo desde 1780 (Barcelona: Edición Crítica, 1991).

${ }^{4}$ Leonard Tivey, El Estado Nación (Barcelona: Nova Grafic, 1987), 25-54.

${ }^{5}$ Ernest Gellner, Naciones y nacionalismos, (Madrid: Alianza 1988), 347.

${ }^{6}$ En el proceso de modernización del Estado hay que tener en cuenta los antecedentes históricos y sociales del siglo XIX. Ver Xosé Manoel Núñez Seixas, Los nacionalismos en la España contemporánea siglos XIX y XX (Barcelona: Hipòtesi, 1999). Respecto a la tesis de débil nacionalización, desarrollada sobre todo en la década de los 90 señalaremos las siguientes obras: Borja de Riquer: Escolta Espan-
} 
castellano o en francés, la cultura y la lengua vasca han perdurando hasta la actualidad, sobre todo en las zonas rurales donde el euskera era lengua vehicular y la única que conocían sus habitantes. No podemos olvidar que la construcción identitaria vasca, en el ámbito escolar, estará limitado por razones sociolingüísticas —el euskara no tiene la misma presencia en todos los territorios-, y por la presencia de una administración y escuela española que se fue extendiendo. ${ }^{7}$

Así, el Estado español nacido del conflicto Tradición-Modernidad será el encargado de redefinir las relaciones con las fuerzas tradicionalistas y vasquistas a finales del siglo XIX que, además de defender su lengua y su cultura, empezará a reclamar algún tipo de institucionalización política del territorio habitado. ${ }^{8}$ En esta época, por lo que se refiere a la construcción de la identidad vasca se puede hablar de dos visiones: una visión en la que el fuerismo y el carlismo vendrían a configurarse como los antecedentes que explicarían el surgimiento del nacionalismo vasco y otra que señala que el discurso fuerista es la versión vasca del nacionalismo español. ${ }^{9}$

En este contexto, la autonomía administrativa y la modernización del sistema educativo serán factores claves en la transformación social y económica del país. Obtener mayores tasas de alfabetización se convertirá en un elemento clave en el proceso de modernización y la escuela será su principal agente como elemento de homogeneización cultural y lingüística, tanto por el Estado como por el nacionalismo vasco.

La llegada del siglo xx trajo consigo, en el Estado español, notables transformaciones sociales, culturales y educativas. En el marco legislativo-educativo del Estado podemos señalar que fueron hitos reseñables: la creación del Ministerio de Instrucción Pública y de Bellas Artes, en 1900; la centralización y garantía de los salarios a los maestros de escuela primaria en 1901; y la ampliación de la escolarización obligatoria hasta los 12 años en 1901. En este proceso de renovación educativa, la zona rural fue la gran olvidada. Así, la falta de escuelas rurales dignas era una realidad

\footnotetext{
ya. La cuestión catalana en la España liberal (Marcial Pons, Madrid, 2002) y la obra de obra de José Álvarez Junco, Mater Dolorosa. La idea de España en el siglo XIX (Taurus, Madrid, 2001).

${ }^{7}$ Paulí Dávila, «Euskal Herria tiene forma de corazón», Historia de la educación, 27 (2008), 215-243.

${ }^{8}$ Francisco Letamendia, El hilo invisible (Bilbao, EHU-UPV, 2013), 302-303.

${ }^{9}$ Coro Rubio, La identidad vasca en el siglo XIX. Discurso y agentes sociales (Madrid, Biblioteca Nueva, 2003).
} 
sangrante en todo el Estado español, y las pocas escuelas existentes o estaban abandonadas a su suerte o se imponía un modelo pedagógico de corte urbano, que no tenía en cuenta la realidad sociocultural del mundo rural. ${ }^{10}$

El proceso de alfabetización en castellano, desarrollado desde finales del siglo XIX, supuso un duro proceso para la mayoría de los niños y las niñas del País Vasco, sobre todo los de las zonas rurales. Esta realidad era bien conocida por el inspector J.M. Eguren, que en sus visitas por las escuelas de Gipuzkoa y Álava de 1859 a 1876, trabajó con ahínco para imponer el castellano en las escuelas y también fuera de ellas. ${ }^{11}$ Dicho inspector reconocía que la masa general del pueblo vasco hablaba habitualmente el vascuence $^{12} \mathrm{y}$, por consiguiente, ésta era la primera lengua que aprendían los niños y las niñas - situación que deseaba cambiar de una forma particular, como señalaba en la introducción de su famoso método-.${ }^{13}$ Esta situación era especialmente relevante en las zonas rurales, con una población exclusivamente monolingüe vasca, euskaldun, y con una población dispersa en barrios alejados de los núcleos urbanos y en caseríos diseminados. En estos barrios las carencias educativas eran mucho mayores que en las zonas urbanas lo que repercutirá en sus tasas de escolarización y alfabetización. ${ }^{14}$

Con la expansión de la revolución industrial y la unificación de los mercados, la alfabetización de la población fue una de las prioridades de

\footnotetext{
${ }^{10}$ Jose María Hernández Díaz, "La escuela rural en la España del siglo xx», Revista de Educación, núm. Extraordinario (2000): 113-136; Paulí Dávila (coord.), Las políticas educativas en el País Vasco durante el siglo XX (Madrid: Biblioteca Nueva, 2004).

${ }^{11}$ Paulí Dávila, y Ana Eizagirre, "Alfabetización y euskaldunización en Euskal Herria», en Leer y Escribir en España, doscientos años de alfabetización, coord. Agustín Escolano (Madrid: Fundación Sánchez Ruiperez, 1992),195.

${ }^{12}$ Según Larronde, quien cita las estadísticas de 1876 de Ladislao de Velasco, la cantidad y la densidad de los vasco-parlantes sería la siguiente: «Guipúzcoa, 170.000 de 176.000 habitantes (140.000 lo hablan habitualmente); Vizcaya, 149.000 de 183.000 habitantes (hay 28.000 personas en el distrito de Valmaseda-Encartaciones que no hablan vascuence, a las que hay que añadir 6.000 extranjeros que residen en Vizcaya: a fines de siglo, esta última cifra será mucho más elevada como consecuencia de la inmigración obrera atraída por el desarrollo industrial); Álava, 12.000 de 120.000 habitantes (esta provincia, profundamente «desvasquizada» desde hace mucho tiempo, no cuenta más que con algunos núcleos vascófonos en las zonas limítrofes con Vizcaya: Aramayona, Villarreal, Llodio); según Ladislao Velasco, hay, pues, en 1867331.000 personas que hablan euskera, de 479.000, en estas tres provincias». Jean-Claude Larronde, El nacionalismo vasco: su origen y su ideología en la obra de Sabino Arana-Goiri (San Sebastián: Ediciones Vascas, 1977), 132.

${ }^{13}$ Paulí Dávila, La política educativa y la enseñanza pública en el País Vasco (1860-1930) (San Sebastián: Ibaeta Pedagogía, 1995), 88-92.

${ }^{14}$ Castells, Modernización y dinámica política en la sociedad guipuzcoana de la restauración (18761915), 14.
} 
las autoridades, tanto en la zona urbana como en las zonas rurales, población que en el caso de Gipuzkoa y Bizkaia, eran mayoritariamente vascoparlante. ${ }^{15}$ Así, eran miles los niños y niñas que no acudían a la escuela, por la distancia en que estaban las escuelas o sólo lo hacían por temporadas, debido a que se ocupaban de los trabajos de campo. Teniendo en cuenta que la escuela pública no era capaz de llegar a muchas zonas rurales, para solucionar, entre otros, el problema de la distancia, se recurrió a la creación de escuelas particulares, las denominadas escuelitas rurales.

Estas escuelitas fueron creadas en la mayoría de los casos a iniciativa de padres, particulares, instituciones locales y sobre todo los curas de los barrios rurales. Uno de los objetivos de estas escuelas era que la enseñanza de la Doctrina Cristiana se diese en vascuence. La mayoría de estas escuelas estaban regidas por maestras y maestros sin título, curas, sacristanes, seroras (mujeres que también a dedicaban a cuidar la Iglesia), que impartían la docencia en euskera, que era la lengua vehicular de los niños y niñas que acudían a estas escuelas. La línea educativa de estas escuelitas solía estar, en la mayoría de los casos, determinada por el cura del barrio, la mayoría de ellos de ideología tradicionalista y euskaldun, en la línea de la Iglesia de la época. Aunque en la mayoría de estas escuelitas no se aprecia una apuesta política nacionalista, en la postguerra la represión castigó también a los maestros de estas escuelas que se identificaron con la causa nacionalista.

En Gipuzkoa, en 1920 el número de escuelitas, escuela rurales particulares incompletas, que se contabilizaban era de $73 .{ }^{16}$ En 1930, en las barriadas rurales funcionaban 74 escuelas rurales subvencionadas, a las que acudían 3.666 niños y niñas. ${ }^{17}$ En los barrios en los que no había escuelas, la Diputación de Gipuzkoa recurrió también al cuerpo de mi-

${ }^{15}$ Pero hay que tener en cuenta, como señala Paulí Dávila, que esta relación alfabetización- industrialización no se corresponde necesariamente en todos los casos. Así en el censo de 1930, Álava - provincia agrícola - figuraba en primer lugar en el ranking de los alfabetizados, con un 22,48\% de analfabetos, seguida de Gipuzkoa con 25,51\%, Bizkaia con 27,31\% — provincias industriales-y Navarra con un 29,24\%. Paulí Davila, Lengua escuela y cultura. El proceso de alfabetización en Euskal Herria, siglos XIX y XX (Donostia, EHU, 1998), 80.

${ }^{16}$ Archivo General de Gipuzkoa, (AGG), situada en Tolosa (Gipuzkoa). (JD IT 1874).

${ }^{17}$ AGG (JD ITF 1460/2056). Inspección de 1934, Escuela del barrio de Arzallus, Errezil. Todavía en algunos caseríos se llama eskola a la habitación en la que, a principios del siglo xx, se impartía la enseñanza. Así lo han podido corroborar los autores de artículo en una entrevista realizada en el año 2003 en el caserío Sarramendi, del barrio de Larrañe de Oñati (Gipuzkoa). 
keletes, ${ }^{18}$ y así estableció escuelitas en algunos de los puestos de mikeletes a petición de los vecinos de distintos barrios rurales. ${ }^{19}$ Pero el mantenimiento y creación de estas escuelitas no era suficiente para solucionar el problema de la enseñanza rural.

Así pues, por lo que respecta al modelo de alfabetización vasco, es preciso señalar las siguientes particularidades: la introducción del castellano como lengua de aprendizaje escolar y consecuentemente de alfabetización, la importancia del régimen municipal en el sostenimiento de las escuelas y la utilización del catecismo en euskara como medio de alfabetización. Ante esta realidad, extender la enseñanza en las zonas rurales se convirtió en objetivo prioritario para las Diputaciones de Bizkaia y de Gipuzkoa.

Tabla 1. Tasas de analfabetismo en el Estado español y en el País Vasco (1900-1920)

\begin{tabular}{|c|c|c|c|c|c|c|c|c|c|}
\hline \multirow{2}{*}{ Año } & $\begin{array}{c}\text { Estado } \\
\text { español }\end{array}$ & \multicolumn{2}{|c|}{ Álava } & \multicolumn{2}{c|}{ Gipuzkoa } & \multicolumn{2}{c|}{ Bizkaia } & \multicolumn{2}{c|}{ Navarra } \\
\hline & Proporción & Población & Proporción & Población & Proporción & Población & Proporción & Población & Proporción \\
\hline 1900 & $63,7 \%$ & 33.054 & $34.2 \%$ & 90.594 & $46,2 \%$ & 145.553 & $46,7 \%$ & 137.221 & $44,6 \%$ \\
\hline 1910 & $59,3 \%$ & 31.456 & $32,3 \%$ & 92.222 & $40,6 \%$ & 142.733 & $40,7 \%$ & 135.539 & $43,4 \%$ \\
\hline 1920 & $52,2 \%$ & 27.064 & $27,4 \%$ & 90.419 & $34,9 \%$ & 133.970 & $32,7 \%$ & 121.332 & $36,7 \%$ \\
\hline
\end{tabular}

Fuente: Paulí Dávila: La política educativa y la enseñanza pública en el País Vasco (1860-1930) (San Sebastián: Ibaeta-Pedagogía, 1995), 188.

En la encuesta realizada por la Diputación de Gipuzkoa en 1914 se señalaba la desoladora situación de la enseñanza. ${ }^{20}$ En 1921, después de

\footnotetext{
${ }^{18}$ Joxe Garmendia, «Mikelete postuetako eskolak», Hik Hasi 62, (2001): 41-43. Desde fines del siglo XIX hasta 1936, los Mikeletes de Gipuzkoa, fueron un grupo armado que realizó tareas administrativas y labores de guarda de las fronteras. La Diputación desarrollo un plan para la implantación de escuelas rurales en los puestos de miqueletes y aunque este plan no llegó a desarrollarse en los términos señalados, sí funcionaron 5 escuelas rurales.

${ }^{19}$ AGG (JD IT 1457, 2). Sobre la petición de abrir escuelas en las zonas rurales. El 17 de septiembre de 1924, el Teniente Coronel Jefe de Mikeletes de Gipuzkoa de la Comandancia n. ${ }^{\circ} 381$ de San Sebastián, señala que «en diversas ocasiones algunos padres de familia se han acercado a los puestos de Miqueletes establecidos en lugares aislados de los poblados solicitando sus servicios para que en ratos perdidos y sin desatender el cumplimiento de las obligaciones que la Provincia les tiene encomendados enseñaran a sus hijos a leer, escribir y otras materias». Aunque se elaboró un plan que contemplaba el establecimiento de 14 escuelas a cargo de los miqueletes, solo se abrieron 5 escuelas.

${ }^{20}$ AGG (JD IT 1455). De esta manera justificaban los ayuntamientos las bajas tasas de alfabetización y escolarización: 1.- El poco entusiasmo y abandono de los padres; 2.- La ocupación en el trabajo, en las labores agrícolas y la pesca; 3.- La distancia de los caseríos y las dificultades topográficas; 4.- La
} 
analizar las respuestas de los ayuntamientos, se subraya la cuestión de la lengua y la presencia del profesorado foráneo como causas de la deficiente situación educativa. ${ }^{21}$ En sus informes las instituciones locales señalaban la incoherencia de que se impartiese la clase en castellano y la dificultad que tenían muchos alumnos de expresarse en castellano:

Lo apropiado es que los maestros posean el idioma vascongado, mejor que sean vascongados y que los nombramientos los haga la Diputación provincial o que los maestros fuesen nominados por los Ayuntamientos por los beneficios que reportaría esto. Así se evitaría que los maestros se encontrasen abandonados y mejoraría la enseñanza. En los pueblos completamente vascos, es absurdo aprender todo en castellano, se necesitan textos en vascuence. $^{22}$

Los padres se quejan de que el maestro carece de lengua vasca y no les enseña la doctrina en dicha lengua y además a los niños por carecer de lengua castellana no le atienden al maestro, aunque este ponga su buena voluntad. Esta es la causa por la que la mayoría asiste a la escuela particular de Cestona. Necesidad de una maestra, en vez de un maestro, con la condición de que sepa el euskera. ${ }^{23}$

La inspección también era consciente del desconocimiento del lenguaje castellano por la casi totalidad de los niños que asistían a estas a las escuelas rurales: ${ }^{24}$

\footnotetext{
poca calidad de la enseñanza: las deficiencias del profesorado, los cambios frecuentes de profesorado y largas vacantes y la falta de maestros titulares; 5.- El problema de la lengua, en algunos casos se subraya la timidez de los niños para presentarse ante maestros que ignoran la lengua vascongada Observaciones de los Ayuntamientos de Ataun, Berastegi, Zegama, Segura, Usurbil, Amezketa, Zegama, Zestoa, Aretxabaleta, Astigarraga, Getaria, Antzuola, Aretxabaleta, Ataun, Aia, Legazpia, Elgeta, Orio, Soraluce, Villabona, Elgeta, Hernani, Errenteria, Eskoriatza, Asteasu.

${ }^{21}$ Dávila, La profesión del magisterio, 111-112.

${ }^{22}$ AGG (JD IT 1874). Observación de los Ayuntamientos de Ibarra, Eskoriatza, Lazkao y Leaburu, 17 de diciembre de 1920.

${ }^{23}$ AGG (JD IT 1874). Observación del Ayuntamiento de Aizarnazabal, 1920.

${ }^{24}$ Archivo General de la Administración (AGA), Madrid. «Memoria del inspector Sanz Rahora, L. de 1906, sobre Gipuzkoa», Leg. 6202: «El desconocimiento del lenguaje castellano por la totalidad de los niños que asisten a las escuelas rurales hace preciso emplear procedimientos intuitivos para la enseñanza de aquél, como base de todos los conocimientos que el niño puede adquirir. Tales procedimientos exigen material adecuado que facilita la labor instructiva del Maestro».
} 
La instrucción es primitiva y aunque tienen algunos textos en castellano, solamente es para saber leer, pues toda la enseñanza la hacen en vascuence (barrio de Urkia).

Aunque la instrucción es en euskara se encuentran muy adelantados (barrio de San Esteban). ${ }^{25}$

Incluso la Comisión de Enseñanza de la Diputación corroboraba que era un absurdo pedagógico el que se diese la enseñanza en una lengua no conocida por los que habían de recibirla. ${ }^{26}$

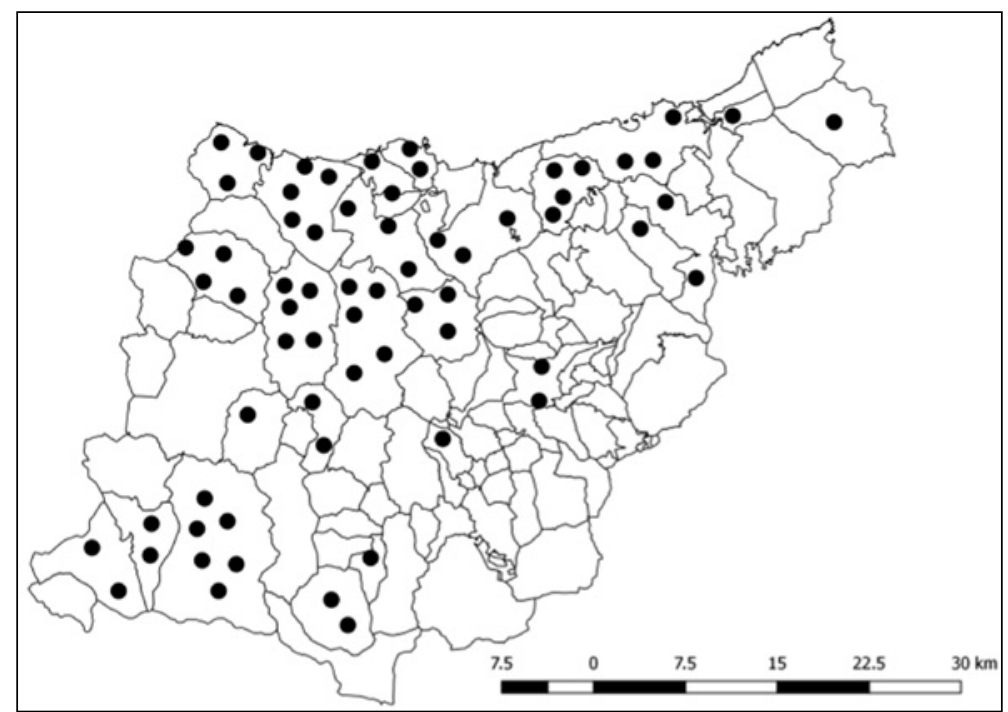

Mapa 1. Escuelitas rurales particulares subvencionadas en Gipuzkoa (1936)27

La realidad educativa de Bizkaia no se diferenciaba de la realidad guipuzcoana. La mayoría de los docentes vizcaínos destacaban en sus memorias anuales el grave problema que suponía que los alumnos no

\footnotetext{
${ }^{25}$ AGG (JD ITF 1460/2054). Inspección de 1932.

${ }^{26}$ Acta de la sesión de la Diputación de Gipuzkoa de 4 de agosto de 1921: «En el territorio vasco son bastantes los maestros que no poseen el vascuence y otros que no lo dominan en el grado de perfección que se requiere para educar e instruir a los niños vascongados. En los muchos pueblos de Guipúzcoa donde los niños no poseen otro idioma que el vascuence por no haber oído hablar otra lengua en sus hogares, verse sometidos a la tortura de tener que recibir las lecciones de su maestro en un idioma que le es completamente desconocido».
}

${ }^{27}$ Garmendia, La enseñanza rural en Gipuzkoa, 207-208. Mapa realizado por los autores. 
conociesen la lengua castellana, lengua en que se expresaban la mayoría del magisterio vizcaíno. En Bizkaia eran muchos los maestros y maestras que, sin saber ni entender euskera, llegaban a las escuelas enclavadas en zonas rurales, donde la población era mayoritariamente vascófona. ${ }^{28}$ Belaustegigoitia, nacionalista moderado, señala que «la mayoría de las escuelas que podemos ver hoy son perjudiciales para el euskera; tanto las del gobierno enemigo, de creencias tibias, como aquellas escuelas creadas entre nosotros, ya sea por quien vista de rojo, o incluso de sotana». ${ }^{29}$

Hay que destacar, también, que la escuela se diseñaba para el ámbito urbano y en sus contenidos aparecía como un elemento extraño para el mundo rural, ya que no tenía en cuenta las peculiaridades del lugar ni la realidad socio-económica. El idioma representaba una primera barrera, la mayoría de los niños desconocía el castellano y el número de maestras y maestros euskaldunes (conocedores del idioma vasco) era insuficiente. ${ }^{30}$ En este contexto se puede entender que gran parte de los ayuntamientos que reivindicasen una mayor implicación de los poderes provinciales en la gestión de la enseñanza.

Con los nuevos cambios sociales y con la implantación de una escuela ajena a la realidad social del País Vasco, la comunidad vasca era cada vez más consciente que las señas de identidad, las tradiciones y la lengua, estaban siendo erosionadas por las nuevas ideologías liberales. Así en el plano educativo, empieza a emerger un movimiento vasquista que reacciona en defensa de la lengua, la religión y la cultura, movimiento que se ven apoyado por algunas fuerzas políticas locales y provinciales.

\section{RENOVACIÓN EDUCATIVA Y ESCUELAS RURALES: LAS ESCUELAS DE BARRIADA Y LAS ESCUELAS PROVINCIALES}

En el primer tercio del siglo Xx, se produjo uno de los hechos culturales más importante de esta época, la creación de la Sociedad de Estudios

\footnotetext{
${ }^{28}$ Ander Delgado, «¿Amantes de Dios de la patria y de la familia?», Educatio siglo XXI, 34 (3), (2016), 47-64.

${ }^{29}$ Iñaki Zabaleta, «La enseñanza en los orígenes del nacionalismo vasco: la cuestión religiosa», en Las políticas educativas en el País Vasco durante el siglo xx, ed. Paulí Dávila (Madrid: Biblioteca Nueva, 2004), 134.

${ }^{30}$ Cándida Calvo Vicente, «La enseñanza en la zona rural guipuzcoana en el primer franquismo», Vasconia, 27, (1998): 166.
} 
Vascos. Así, carlistas, euskalerríacos, liberales y nacionalistas promovieron por primera vez una entidad de carácter supraprovincial en la que colaboraron, además de las diputaciones de Bizkaia, Gipuzkoa, Araba y Navarra, los Obispos de Vitoria, Pamplona y Bayona. A partir de 1918 se celebraron los Congresos de Estudios Vascos. ${ }^{31}$ En ellos que se reivindicó, entre otras aportaciones, una enseñanza acorde a la realidad del País en cuanto a la lengua y a la cultura. ${ }^{32}$ En los primeros Congresos (Oñati, 1918, Pamplona, 1920, y Gernika, 1922) la presencia de los temas relacionados con la enseñanza fue constante. En el Primer Congreso, en 1918, se analizó básicamente la historia y la situación de la enseñanza primaria. En este Congreso hay que destacar las aportaciones de Eduardo Landeta, Adelina Méndez de la Torre, Leoncio de Urabayen y Luis de Eleizalde, ${ }^{33}$ que presentaron la realidad educativa del País, así como las propuestas para mejorarla. El Segundo Congreso de Estudios Vascos se celebró en 1920 en Pamplona, y estuvo dedicado a la «Enseñanza y cuestiones económico sociales». ${ }^{34}$

Pero no debemos de obviar, como antes se ha señalado, que en los barrios rurales, a falta de otras escuelas el modelo más extendido a principios del siglo xx era el de las escuelitas rurales. ${ }^{35}$ Estas escuelas creadas por iniciativa de los padres recibían, en muchos casos, una pequeña ayuda económica de los ayuntamientos y diputaciones.

\footnotetext{
${ }^{31}$ Idoia Estornés, La Sociedad de estudios vascos. La aportación de Eusko Ikaskuntza a la cultura vasca (1918-1936) (San Sebastián: Eusko Ikaskuntza, 1983).

${ }^{32}$ Garmendia, La enseñanza rural en Gipuzkoa, 148-157.

${ }^{33}$ Luis de Eleizalde, escritor, pedagogo y político guipuzcoano (Bergara, 1878- Bilbao, 1923). Inspector de Enseñanza de la Diputación Bizkaia. Fue miembro fundacional de Euskaltzaindia. Entre sus obras más conocidas podemos citar: Razas, lengua y nación vascas, 1911; Morfología de la conjugación vasca sintáctica, 1913; Metodología para la restauración del euzkera, 1918; Euskal-zenbakistia, 1920 y Euskeraz irakurtzeko irakaspidea.

${ }^{34}$ Estornés, La Sociedad de estudios vascos, 73-91.

${ }^{35}$ Pero esta realidad no era exclusiva del País Vasco; lo mismo ocurría en diversas regiones del Estado español. A principios de siglo, en Galicia, también, las escuelas rurales gallegas eran escasas; los maestros pocos, mal pagados y mal preparados. Los niños dejaban la escuela para trabajar y ayudar a sus familias y los locales seguían siendo como en el siglo XIX, sin material escolar, lúgubres, con poca higiene, sin ventilación, ni iluminación. Así no es de extrañar que los vecinos de las zonas más desfavorecidas intentaran paliar este déficit creando por su propia cuenta y riesgo escuelas privadas que recibieron el nombre de escolas de ferrado. Narciso de Gabriel, Escolantes e Escolas de Ferrado (Vigo, Xerais, 2001); Anton Costa, «Escolas en contornas rurais en Galicia: horizonte difícil/horizonte posible», Innovación Educativa, 24 (2014): 33-153; Blanca Martínez, «La formación de capital humano en Galicia», Revista Galega de Economía, 12 (1), (2003): 1-22.
} 
A partir de estas y otras aportaciones se plantearán diversas propuestas: unas en la línea de trabajar desde fuera de los cauces oficiales o institucionales, otras intentarán trabajar desde el propio sistema escolar oficial. Este último movimiento tendrá una corta vida, al estar condicionada por la coyuntura política. ${ }^{36}$

Todas estas escuelas tenían como elemento distintivo la introducción del euskara en las escuelas. ${ }^{37}$ En el ámbito rural, las nuevas experiencias de las escuelas de barriada y las escuelas provinciales rurales responden a la necesidad de alfabetizar la población rural euskaldun y aportar un carácter renovador a las escuelas rurales.

Superar esta realidad fue el objetivo de las nuevas experiencias de Bizkaia y Gipuzkoa. Con los proyectos citados se edificaron nuevas escuelas, siguiendo las pautas de las nuevas construcciones escolares, se dotó a estas escuelas de materiales innovadores, y se exigió que los maestros y maestras que tuvieran que ejercer en estas escuelas estuvieran titulados y dominaran el euskera. La política educativa de estas escuelas dependió de la correlación de fuerzas políticas. Aunque las escuelas de barriada de Bizkaia fueron promovidas por el nacionalismo vasco, los cambios políticos acaecidos en esta provincia condicionaron su evolución. Por lo que respecta a las escuelas provinciales de Gipuzkoa, a pesar de ser escuelas en las que a sus profesores se les exigía el conocimiento del euskara, era evidente que sus promotores estaban más preocupados por el analfabetismo rural que por promocionar la enseñanza en la lengua vasca. Teniendo en cuenta que la lengua representa la particularidad más específica de una cultura, era evidente que en la zona rural esta se había mantenido porque era la lengua que se utilizaba habitualmente. Como se puede observar, hasta ahora la apuesta de las instituciones y autoridades políticas por una educación en euskara había sido muy débil, el

${ }^{36}$ Paulí Dávila, «Curriculum vasco, agentes sociales y libros de texto en euskara hasta 1937», Euskonews, 265 (2008): 12-19; consultado el 11 de enero 2017. URL: http://www.euskonews.com/0465zbk/ gaia46504es.html.

${ }^{37}$ Trabajando desde fuera de las instituciones, una de las experiencias que se desarrolló en un ámbito urbano fue el modelo de Escuela Vasca. Este proyecto es una experiencia de escolarización exclusivamente en euskara, que se enmarca dentro del proyecto nacionalista, y que será referente para las ikastolas que se crearán durante el franquismo. En esta experiencia la escuela es concebida como medio para concienciar a los alumnos vascoparlantes, desde la perspectiva del nacionalismo vasco. 
bilingüismo diglósico estaba a favor del castellano. ${ }^{38}$ Por lo que respecta a la metodología educativa, en estas nuevas escuelas hay que reseñar que se tenía en cuenta la pedagogía moderna y el material era sencillo pero novedoso. ${ }^{39}$

\section{Las Escuelas de Barriada de Bizkaia}

La presencia de los nacionalistas en la Diputación de Bizkaia, a partir de 1917, y unas condiciones políticas favorables, hicieron posible que prosperase esta interesante experiencia, tanto por la intervención de las instancias públicas como por su aportación pedagógica. ${ }^{40}$ Este intento no era el primero, en 1896 lo había intentado el diputado Victoriano Gáldiz y en 1911 el diputado Isidoro León, pero estas propuestas no prosperaron. ${ }^{41}$ A partir de 1917, con la creación de la Junta de Instrucción Pública y la Junta de Cultura de la Diputación de Bizkaia, se fue afianzándose la idea de la escuela vasca. En esta la Junta de Instrucción participaron personas tan cualificadas como, E. Landeta, L. Eleizalde y el mismo Gallano. ${ }^{42}$ Así, la Diputación de Bizkaia trataba de extender la instrucción primaria y que esta fuera en euskara:

Todos sabéis que en la parte rural de Bizkaia hay una masa muy importante de población joven que no conoce el castellano, que habla simplemente el euskera, a esa juventud se le enseñan las primeras letras en castellano. Y resulta que como no entienden lo que se les enseña, salen de las escuelas sin saber leer ni

\footnotetext{
${ }^{38}$ Paulo Iztueta, «Las contradicciones de la cultura vasca: Teoría y praxis», en Lengua, escuela y cultura. El proceso de alfabetización en Euskal Herria, siglos XIX y XX, coord. Paulí Davila (Bilbao: Universidad del País Vasco, 1995), 349-50.

${ }^{39}$ Gregorio Arrien, Educación y escuelas de Barriada en Bizkaia. Escuela y Autonomía 1898-1936 (Bilbo, Bizkaiko Foru Aldundia, 1987), 165-175.

${ }^{40}$ En mayo de 1917, tras las elecciones provinciales, se constituyó en Bizkaia la nueva Diputación provincial. El nuevo Presidente de la Corporación, Ramón Sota, había sido alumno de Azkue y alumno del King's College de Londres, respecto a la educación rural señalaba lo siguiente: «En la parte rural de Vizcaya hay una masa muy importante de población joven que no conoce el castellano, que habla simplemente el euskara; a esa juventud se le enseñan las primeras letras en castellano. Y resulta que como no entienden lo que se les enseña, salen de las escuelas sin saber leer ni escribir, es decir, han perdido el tiempo» Archivo Administrativo Diputación de Vizcaya: Sesión de Diputación de 4-V-1917 (BOPV de 23-V-1917, n. ${ }^{\circ}$ 114), 494.

${ }^{41}$ Maite Paliza, El proyecto de las escuelas de barriada su desarrollo (1919-1938) Una epopeya de la historia de la educación en Bizkaia (Bilbao: Gobierno Vasco, 2015), 33.

${ }^{42}$ Arrien, Educación y escuelas; Dávila, La política educativa, 167-168.
} 
escribir, es decir que han perdido el tiempo. Es preciso que a esta parte de nuestra población se le enseñe en euskera, naturalmente que para ello tendremos que recabar del Estado una libertad absoluta de enseñanza conjuntamente con las otras Diputaciones. ${ }^{43}$

El 26 de noviembre de 1919 se presentó la moción que llevaba el nombre del diputado provincial encargado de presentarla, el diputado Gallano, con el objeto de crear centros de educación e instrucción para reducir los altos niveles de analfabetismo y corregir los desajustes educativo-culturales de la provincia, sobre todo de las zonas rurales y de los barrios minero-industriales. El proyecto de las escuelas de barriada de Bizkaia trajo consigo la reducción del analfabetismo y la instauración de una educación bilingüe. Así mismo, con esta iniciativa se intentó mejorar el nivel de vida de los habitantes de las zonas rurales y costeras, zonas de gran relevancia para el nacionalismo. La labor educativa de estas escuelas mereció toda clase de elogios, así la Comisión de Primera Enseñanza de Estudios Vascos señalaba lo siguiente:

Una de las más grandiosas iniciativas que cabe esperar de una administración pública bien orientada al aproximar los centros de enseñanza a los más apartados lugares de población. Estas diputaciones vascas han servido un ejemplo práctico de lo que de construir el ideal de nuestro pueblo: el mantenimiento de su organización nativa y característica y el mejorar dentro de ese sistema natural su situación cultural. No son obstáculos a vencer la peculiar, natural y recomendable diseminación de la población, ni la existencia de un idioma propio de la tierra. No son obstáculos, no son inconvenientes que haya que subsanar; son valores que posee el País Vasco, que hay que conservar, proteger y fomentar, creando soluciones adecuadas a una organización propia y no alterando ésta a la medida de fórmulas comunes preestablecidas. $^{44}$

\footnotetext{
${ }^{43}$ Boletín Oficial de la Provincia de Vizcaya, 23 de mayo de 1917.

${ }^{44}$ Arrien, Educación y escuelas, 196.
} 
La construcción de las Escuelas de Barriada estuvo a cargo del arquitecto provincial Diego Basterra (1883-1959). ${ }^{45}$ En el diseño de este proyecto participó la Sociedad de Estudios Vascos que, entre otras peticiones, reivindicaba la implantación de la enseñanza en euskera desde párvulos, la edición de libros de texto y material en ese idioma, y la incorporación de la asignatura de Geografía e Historia del País Vasco. ${ }^{46}$

Así pues, en la evolución del presupuesto de la Diputación de Bizkaia durante estos años, se aprecia un fuerte aumento en la inversión educativa. Mediante este proyecto la Diputación vizcaína propuso:

La creación de cincuenta Escuelas primarias de niños y cincuenta de niñas en un periodo de cinco años, en otras tantas barriadas o agrupaciones de más de diez familias cuyas viviendas disten por lo menos un kilómetro de la Escuela más próxima, gestionando al efecto la cesión gratuita del local más adecuado que dispongan los vecinos favorecidos y costeando la Excma. Diputación el material de enseñanza necesario y el sostenimiento de los Maestros, cuyo sueldo mínimo será de 3.000 pesetas. Consignación de la cantidad de 90.000 pesetas en los presupuestos del próximo ejercicio para la creación de las veinte Escuelas correspondientes al primer año. ${ }^{47}$

Las escuelas de barriada fueron concebidas, en sus orígenes, como verdaderas escuelas vascas. Este proyecto pretendía desarrollar un proyecto bilingüe desde las estructuras del propio sistema escolar y oficial, impulsado por destacados personajes nacionalistas como Landeta y Eleizalde. Como señala Zufia, la Diputación se encontraba ante el problema del vascuence y hacer caso omiso a él era tanto como llevar de antemano al fracaso a estas escuelas. No podía exigirse al maestro una labor eficaz

\footnotetext{
${ }^{45}$ Paliza, El proyecto de las escuelas de barriada, 84. Diego Basterra, de ideología nacionalista fue concejal del Ayuntamiento de Bilbao por ese partido. Alcanza la condición de funcionario del Ayuntamiento de Bilbao en 1919 y permaneció al frente del Servicio de arquitectura hasta 1937, cuando fue detenido y encarcelado hasta 1940. Por lo que se refiere a las escuelas de barriada, diseño los prototipos, inspeccionó las obras y autorizó su apertura o clausura. Aunque trabajó prácticamente en solitario, contó con la ayuda del delineante del Servicio de Arquitectura de la Diputación, Luis Elejabeitia (nacido en Deusto en 1889).

${ }^{46}$ Garmendia, La enseñanza rural en Gipuzkoa, 154-155.

${ }^{47}$ Zufía, Las Escuelas de Barriada en Vizcaya, (Bilbao: Imp. Provincial, 1930).
} 
si teniendo que actuar en las zonas rurales - en las que, como se ha recalcado, solo se hablaba el euskara- no conocía esa lengua.

Este proyecto sufrió importantes modificaciones a lo largo del periodo 1919-1936. Como señala Dávila, con la aparición de la Liga Monárquica, fuerza surgida con el objetivo de romper la hegemonía nacionalista y que ya en 1919 había conseguido éxitos importantes, las buenas perspectivas iniciales se verían truncadas. ${ }^{48}$ Así, a partir de 1921, con la Liga Monárquica en la Diputación de Bizkaia, la oposición al euskera se incrementó notablemente. En las escuelas de barriada fueron suprimidos los textos publicados en euskera y que se estaban utilizando. ${ }^{49}$ Para la Liga de Acción Monárquica lo único que pretendían los nacionalistas era propagar el uso del vascuence, y que los que lo hablaban desde niños no llegasen a aprender el castellano.

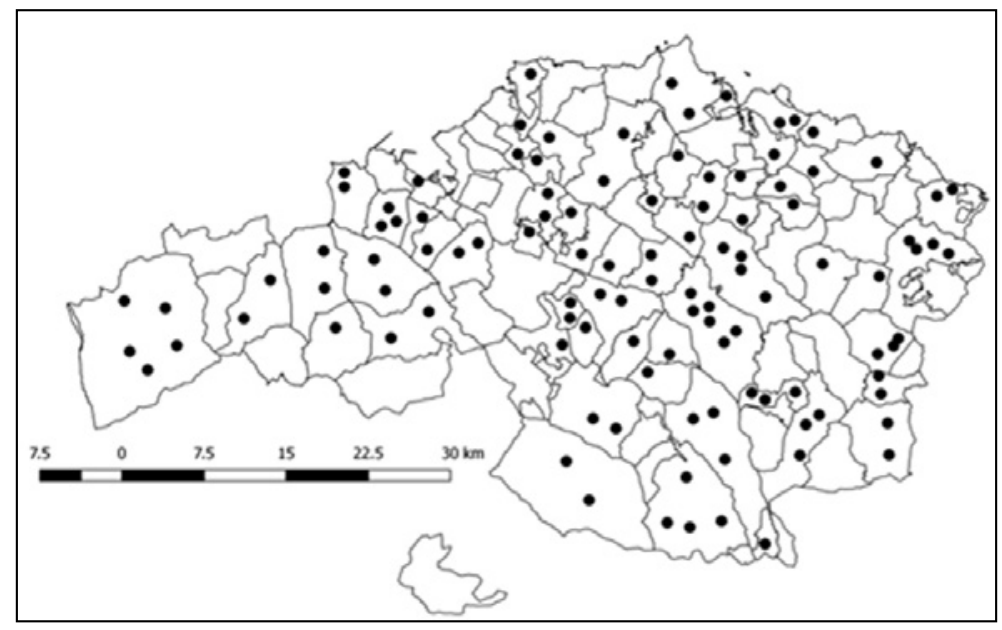

Mapa 2. Escuelas de Barriada de Bizkaia (1921-1934) ${ }^{50}$

En el proyecto inicial de estas escuelas de Barriada se señalaba que la escuela se adaptaría a las características del medio; así se dividía Bizkaia

\footnotetext{
${ }^{48}$ Dávila, La política educativa, 170.

${ }^{49}$ Paliza, El proyecto de las escuelas de barriada, 39.

${ }^{50}$ Paliza, El proyecto de las escuelas de barriada, 60-62, 66-68. Mapa realizado por los autores.
} 
en tres zonas: marítima, agrícola e industrial. ${ }^{51}$ Teniendo en cuenta el idioma, las escuelas de barriada fueron agrupadas en dos categorías A y B. Categoría A, los que se situaban en la zona vasca, euskaldun; impartían la clase en euskera siendo materia obligatoria el castellano; categoría B, la zona de habla castellana; los maestros/as empleaban el castellano y el euskera era materia obligatoria. ${ }^{52}$

La cuestión del bilingüismo ocasionó diversos problemas a la corporación provincial vizcaína con el Gobierno central y las autoridades académicas de la Universidad de Valladolid, de donde dependían las escuelas del país. Un dictamen del rector de la Universidad, fechado en 1921, exigió que se empleara la lengua y gramática castellana. La Diputación acató la imposición de la autoridad competente; así, obligó a los maestros a utilizar los libros de texto que estaban escritos en lengua castellana y a emplear la lengua vasca solo como medio auxiliar de enseñanza. ${ }^{53}$

La elaboración de textos en euskera, con destino a estas escuelas fue un paso muy importante por lo que respecta a la alfabetización en euskara; así se llegaron a confeccionar multitud de libros, abarcando todas las asignaturas de la enseñanza elemental. ${ }^{54}$ Como se puede observar, se estaba configurando un nuevo modelo educativo, modelo que estuvo condicionado por los avatares y cambios políticos del país. ${ }^{55}$ Toda esta producción de libros de texto ponía de manifiesto la necesidad de construir un currículum vasco más allá de la alfabetización rural. ${ }^{56}$ Cuando en 1922 se prohibieron los textos editados en euskera, solamente sería autorizado el catecismo vasco en las escuelas de la zona A.

\footnotetext{
${ }^{51}$ Paliza, El proyecto de las escuelas de barriada, 191. De los cien edificios que funcionaban en 1930, ochenta y tres estaban en un medio rural, nueve en aéreas industriales, cinco en zonas mineras y uno en el ámbito costero.

${ }^{52}$ Reglamento de Escuelas de Barriada de Vizcaya (Bilbao: Imprenta provincial, 1921), 35.

${ }^{53}$ Paliza, El proyecto de las escuelas de barriada, 65.

${ }^{54}$ Arrien, Educación y escuelas, 210-211. Es preciso citar que el primer libro de lectura en euskara comenzará a gestarse a partir de 1897 cuando Arana-Goiri publica el Umiaren lenengo aizkidea. En los años posteriores las publicaciones son escasas; será a partir de 1918 cuando empiecen a publicarse los libros escolares en euskara.

${ }^{55}$ Arrien, Educación y escuelas 172-173.

${ }^{56}$ Gurutze Ezkurdia Arteaga, «Euskal Herria gaiaren garapena XX. eta XXI. mendeetako testuliburuetan» Ikastaria, 16 (2008): 109-142; Begoña Bilbao, Gurutze Ezkurdia y Karmele Perez, Euskal Curriculuma ala Euskal Dimentsioa Curriculumean? (Donostia, Utriusque Vasconiae, 2004).
} 
En esta experiencia hay que destacar la labor realizada por dos de sus inspectores: L. Eleizalde y P. Zufía, ${ }^{57}$ que ejerció como tal entre los años 1924-1937. Zufía, en su Memoria sobre las Escuelas de Barriada de Bizkaia, señalaba que transcurridos 8 años se podía afirmar que con las cien escuelas construidas el plan inicial estaba terminado. ${ }^{58}$

El desarrollo de la guerra condicionó el funcionamiento de estas escuelas. Las que se encontraban en el frente de guerra en 1936 fueron cerradas, las demás funcionaron con relativa normalidad hasta abril de 1937. Con el nuevo régimen dictatorial en el poder, su labor fue hacer desaparecer todo lo que podía relacionarse con referencias vascas y republicanas, reprimiendo y suprimiendo todo rastro de sentimiento nacionalista, depurando al profesorado y eliminado toda experiencia de enseñanza en euskera y renovadora. Las nuevas orientaciones académicas del franquismo fueron reafirmando los valores ideológicos del nacional-catolicismo. En un contexto de pobreza generalizada, ni la renovación pedagógica ni la enseñanza en las zonas rurales fue prioritaria para el nuevo orden. La nueva ideología nacional española no podía permitir el rebrote del nacionalismo vasco.

El Decreto-Ley de 23 de junio de 1937 vino a suprimir el Régimen del Concierto Económico para Bizkaia y Gipuzkoa, con lo que despareció sin más la base económica para el sostenimiento de estas escuelas. Por la Orden de 24 de noviembre de 1937, todas las escuelas de barriada y provinciales creadas por estas Diputaciones, pasaron a depender del Estado. Esta Orden fue completada por la de 18 de abril de 1938, que reglamentaba lo referente al personal docente, sus retribuciones, sus derechos y obligaciones. ${ }^{59}$

\section{Las Escuelas provinciales de Gipuzkoa (1930-1938)}

La preocupación de las instituciones guipuzcoanas por la enseñanza en el entorno rural se evidencia en las distintas encuestas realizadas a los

\footnotetext{
${ }^{57}$ Paliza, El proyecto de las escuelas de Barriada, 87.

${ }^{58}$ Para conocer cómo eran estas escuelas recurriremos a la descripción que hizo P. Zufía, en la Memoria presentada en 1930. Zufía, Las escuelas de barriada en Vizcaya (Bilbao: Diputación de Vizcaya, 1930).

${ }^{59}$ Orden de 18 de abril de 1938 (BOPV de 22 de abril de 1938, n. ${ }^{\circ}$ 548), 345-346.
} 
ayuntamientos en 1914 y 1920 y en el plan propuesto en $1927 .{ }^{60}$ En un primer momento la Diputación optó por una política de subvenciones a los maestros y maestras que impartían docencia en los diversos caseríos dispersos, pero viendo que esta propuesta era insuficiente, diseñó una nueva propuesta tomando como referencia las escuelas de barriada.

Así, en la sesión de 1 de mayo de 1930 el Sr. Laffitte, Presidente de la Diputación de Gipuzkoa, después de realizar un breve repaso respecto a la paupérrima situación de las escuelas rurales existentes, señaló que los hijos de los caseros no disponían de escuelas necesarias. Así pues, después de esta presentación dio los primeros pasos para la puesta en marcha un proyecto que tenía como objetivo la creación 100 escuelas rurales provinciales. ${ }^{61}$ La aceptación de este Proyecto, en diciembre de 1930, coincidió con un periodo social de gran turbulencia social y política. ${ }^{62}$ A la hora de definir este proyecto, la labor de la Iglesia, los maestros y la utilización del euskera fueron objeto de ardua discusión entre los diputados guipuzcoanos. ${ }^{63}$ Aunque el objetivo inicial fue la construcción de 100 escuelas solo se construyeron 21 en cinco años. ${ }^{64}$ La construcción de estas escuelas de nueva planta, promovida por los municipios y la Diputación, no fue ajena al proceso de modernización que se estaba dando en la enseñanza. ${ }^{65}$

\footnotetext{
${ }^{60}$ Dictamen proponiendo una solución al problema de la enseñanza. Acta de la sesión de la Diputación de Gipuzkoa, 28 de diciembre de 1927.

${ }^{61}$ Acta de la Diputación de Gipuzkoa 1 de mayo de 1930: «Es preciso que a los niños de las barriadas rurales se les atienda de igual forma que a los de las ciudades, dotándolas de cantinas escolares y otros beneficios pues no hay que olvidar que aquellos pobres niños tienen que recorrer grandes distancias para acudir a la escuela».

${ }^{62}$ Emilio Lopez Adán, El Nacionalismo Vasco (1876-1936) (San Sebastián: Txertoa, 1977).

${ }^{63}$ Acta de la sesión de la Diputación de Gipuzkoa, 12 de diciembre de 1930. Entre otras cosas se señalaba que «Se respetara, ante todo, el derecho de la Iglesia, tanto a la hora de crear centros de Instrucción Primaria en las zonas rurales, así como a la hora de inspeccionar las que iba a construir la Diputación. Se critica el sistema antipedagógico que se había seguido hasta ahora, recibiendo las clases en un idioma que no entendían. Pero se subrayaba que a pesar de que se iniciase la enseñanza en euskera, estos tenían que aprender también el castellano, teniendo en cuenta la importancia del mismo en la sociedad; se reivindica el asesoramiento pedagógico de la SEV para desarrollar el proyecto».

${ }^{64}$ Garmendia, La enseñanza rural en Gipuzkoa, 210-211.

${ }^{65}$ Ramón López, «La construcción y creación de escuelas en la España del primer tercio del siglo XX». Historia de la educación, 16 (1997): 65-90.
} 
En este periodo de transformación social y económica, fueron la Diputación y los Ayuntamientos los promotores de este proyecto, siendo su labor complementada con la colaboración de los vecinos. La apuesta por las escuelas rurales fue apoyada no solo por los las instituciones locales y provinciales, sino también por distintos movimientos sociales y agrarios, entre otros por el sindicato agrario Euzko Nekazarien Bazkuna-Asociación de Agrarios vascos, vinculado al nacionalismo vasco. ${ }^{66} \mathrm{El}$ diseño de los nuevos edificios escolares fue obra del arquitecto de la Diputación, Sr. Cortázar.

El 7 de abril de 1931, la Comisión de Primera Enseñanza creada por la Sociedad de Estudios Vascos para conocer y estudiar la situación y necesidades de estas escuelas aprobó las «Bases para la creación y régimen de las Escuelas Rurales de Guipúzcoa», del conde de Villalonga ${ }^{67}$ Estas bases servirán de base para el futuro proyecto de la Diputación. El 16 de mayo de 1931, la misma Comisión, señalaba que era necesario tener en cuenta los valores tradicionales, e impartir la enseñanza en lengua vasca, que era la lengua que la población autóctona utilizaba. Subrayaba la importancia de valorar la cultura vasca que era patrimonio de todos los vascos, y el riesgo de que las escuelas rurales colaborasen en su destrucción. En el mismo documento se criticaba la política escolar de las Diputaciones, señalando el efecto negativo que la actitud seguida por estas instituciones había producido en la mentalidad de los hombres y mujeres de aldea, en los baserritarras (caseros/as), al no potenciar el euskara en la enseñanza:

Que piensa que su idioma propio no le va a servir jamás para nada, que cree que el euskera es inepto para la enseñanza y que por ello desea abandonarlo, para sustituirlo por el castellano. ${ }^{68}$

\footnotetext{
${ }^{66}$ Mikel Aizpuru Murua, «El agrarismo guipuzcoano y el nacionalismo vasco (1920-1933)», Historia Agraria, 70 (2016): 180. Las distintas asociaciones y sindicatos agrícolas Anaitasuna-Hermandad (1900), Alkatasuna-Solidaridad (1905), recibieron el calificativo de católicos y entre otros objetivos tenían el objetivo de hacer frente a la amenaza del socialismo. En 1931 se creó el sindicato agrario Euzko Nekazarien Bazkuna-Asociación de Agrarios vascos, vinculado al nacionalismo vasco, aunque formalmente independiente del mismo.

${ }^{67}$ AGG (JD IT 1874). Información complementaria sobre este proyecto en el Boletín de la Sociedad de Estudios Vascos, Eusko Ikaskuntzaren Deia, 50 (1931) 8-10.

${ }^{68}$ AGG (JD IT 1874), 16 de mayo de 1931.
} 
Y se añadía también que al idioma vasco se le había abandonado históricamente, estando sujeto a las injerencias políticas, restringiendo su uso y deteniendo su desarrollo cultural. Esta Comisión, en sus conclusiones y en la línea de las primeras escuelas de barriada, añadía, entre otras, las siguientes recomendaciones:

- En el ámbito escolar, las escuelas se deberían de clasificar en dos grupos: A) Aquella en la que toda la enseñanza debe darse en el idioma vasco. En este grupo el estudio de la lengua castellana es materia obligatoria en todos los grados o cursos. B) Donde se procede a usar el castellano como idioma general para la enseñanza. El estudio de la lengua vasca es obligatorio en todos los grados. En ningún caso se justificaba un tercer grupo bilingüe.

- Respecto a los libros de texto, las obras escritas en lengua vasca la Diputación debía de tener en cuenta los informes de la Sociedad de Estudios Vascos u otras agrupaciones análogas, tal como la Comisión pro-euskera de la Diputación de Guipuzcoa. ${ }^{69}$

La labor que estaban desarrollando la Diputación y la Sociedad de Estudios Vascos, fueron objeto de las críticas por parte del nacionalismo vasco. Así J. Ariztimuño, Aitzol, ${ }^{70}$ criticó a la Sociedad de Estudios Vascos señalando que, si bien había dedicado buena parte de los primeros Congresos a la enseñanza vasca, su labor sólo se reducía a una aportación teórica. Respecto a los proyectos de las diputaciones y ayuntamientos, señalaba que estos jamás habían prestado una ayuda real al euskara, excepto en momentos y causas concretas (así, las escuelas de barriada de Bizkaia en sus primeros años).

Aunque en la contratación de maestras y maestros la Diputación exigió el conocimiento del euskera, en la contratación de la figura clave, la inspección, no siguió ese criterio. Aunque en un primer momento se pro-

${ }^{69}$ AGG (JD IT 1874).

${ }^{70}$ José de Ariztimuño Olaso (1996-1936). Conocido también por el seudónimo de Aitzol, fue un clérigo, periodista y escritor en euskera y castellano. Aitzol fue fundador de la revista Yakintza y director de la sociedad Euskaltzaleak. Durante la Guerra Civil, fue detenido y fusilado por las fuerzas franquistas a pesar de su condición de sacerdote. Fue un activo miembro del Partido Nacionalista Vasco. Uno de sus libros más conocidos es La democracia en Euzkadi. Lucha de idiomas en Euzkadi y en Europa, editado en 1935 y que ha sido actualmente reeditado por la editorial Erein de Donostia. 
puso dar preferencia a un vascoparlante para esta plaza, ${ }^{71}$ finalmente se eligió como inspectora a la profesora Josefina Olóriz, ${ }^{72}$ que no dominaba el euskera. Esta decisión fue objeto de diversas críticas. ${ }^{73}$

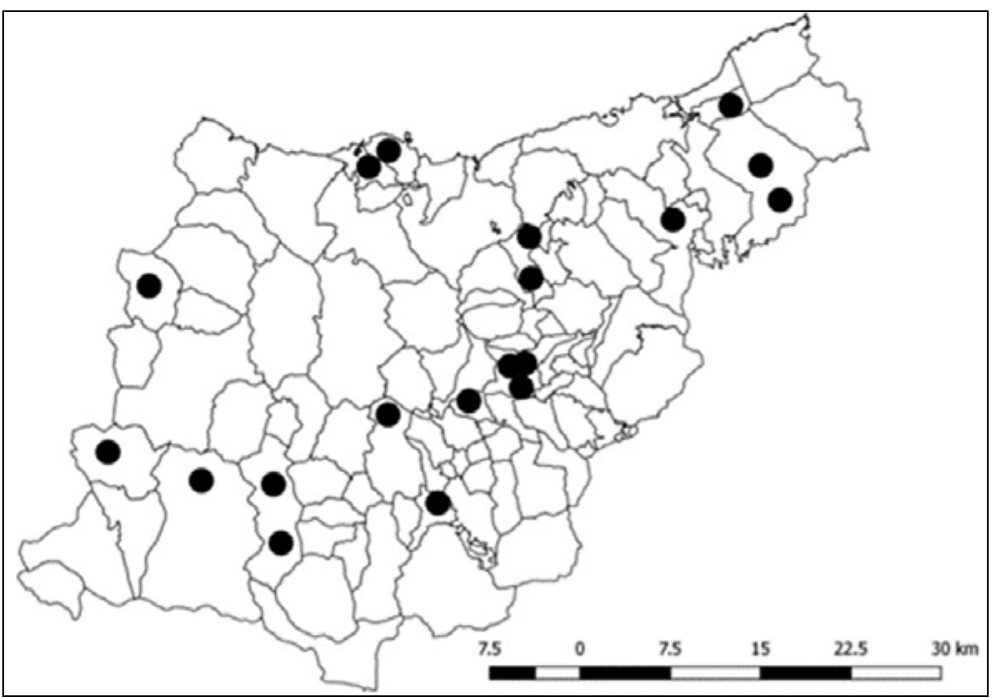

Mapa 3. Escuelas rurales provinciales de Gipuzkoa (1933-1936) ${ }^{74}$

Las primeras escuelas provinciales comenzaron con su labor pedagógica en el curso escolar 1933-34. Por lo que respecta a sus planteamientos

${ }^{71}$ BOG de 13 de febrero de 1931. «Condiciones para la provisión de una plaza de inspector/a: Haber nacido en Guipúzcoa o ser hijo de padres guipuzcoanos [...], entre los que reúnan las citadas condiciones serán preferidos los que conozcan el vascuence».

72 Josefina Olóriz Arzellus, nació en Mar de Plata, Buenos Aires (Argentina), el 27 de agosto de 1894. Hizo sus estudios de primaria en Mar de Plata y cursó la enseñanza media en San Sebastián y en Madrid. Los estudios de magisterio los realizo en San Sebastián, los de carácter superior en Burgos. Ocupó el puesto de profesora catedrática auxiliar en la Normal de San Sebastián de 1914 a 1929 y el de inspectora de primera Enseñanza desde 1929 hasta 1964, año en el que se jubiló. En esta época fue inspectora de las escuelas rurales de la Diputación de Gipuzkoa, nombrada por concurso el 25 de marzo de 1931 y cesó en el cargo el 15 de agosto de 1937. Fue concejala del Ayuntamiento de San Sebastián en 1925 y destacada pedagoga teresiana. Podemos encontrar información sobre esta inspectora en el Archivo General de la Administración (AGA) sig. Top. 32 y el Archivo Administrativo de la Diputación de Gipuzkoa, expediente 3618. Expediente Josefina Olóriz. Para profundizar en la historia de la Institución Teresiana se puede consultar el libro Historia de la Institución Teresiana (1911-1936), de Francisca Rosique, publicada en Madrid, en 1914 por la editorial Sílex.

${ }^{73}$ José Ariztimuño, Obras Completas 2 La democracia en Euskadi. Lucha de idiomas en Euskadi y en Europa. (Donostia: Erein, 1986), 256-262.

${ }^{74}$ Garmendia, La enseñanza rural en Gipuzkoa, 221-222. Mapa realizado por los autores. 
pedagógicos, la inspectora Josefina Olóriz rechazaba categóricamente las lecciones de memoria y el uso excesivo de los libros de texto, recomendando en su lugar la comunicación viva, la interpretación de los fenómenos y las excursiones. El que las maestras y maestros supieran euskera facilitó mucho la enseñanza, pero el objetivo primordial de estas escuelas no fue la enseñanza en euskera, sino que mediante el euskera se potenciase la adquisición del castellano y, a partir del aprendizaje del mismo, profundizar en el conocimiento de los libros escritos en esa lengua.

Este proyecto, que fue potenciado sobre todo en los primeros años de la II República, sufrió un parón en el Bienio Cedista. Posteriormente, el Frente Popular no tuvo tiempo real para poner en marcha las modificaciones necesarias que había acordado. Estas escuelas funcionaron como escuelas provinciales durante cuatro cursos. El plan inicial de construir 100 escuelas en 5 años no se desarrolló, sobre todo por la falta de recursos económicos, y también por la actitud de ciertos ayuntamientos que veían con recelo a estas escuelas. A falta de otra alternativa real, las instituciones provinciales y locales siguieron subvencionando a las escuelitas rurales.

En 1938, como consecuencia de la derogación de los Conciertos Económicos, estas escuelas provinciales pasaron a depender del Estado. El 20 de junio de 1937, funcionaban 21 escuelas, seis de tipo doble (con escuela de niños y escuela de niñas) y las restantes sencillas (escuelas mixtas). Estaba también en tramitación la construcción de otras cuatro para dicho año. ${ }^{75} \mathrm{El}$ golpe militar de 18 de julio de 1936 fue el fin de muchas ilusiones y proyectos, entre otros el de las escuelas provinciales. Con las Comisiones Depuradoras ${ }^{76}$ llego la represión a estas escuelas provinciales y a las escuelitas que todavía funcionaban. ${ }^{77}$ Por la Orden de 24 de noviembre de 1937 las escuelas provinciales pasaron a depender del Estado. Tras la guerra civil, aunque la Diputación guipuzcoana era consciente de que para ejercer en estas escuelas rurales era imprescindible el

\footnotetext{
${ }^{75}$ Garmendia, La enseñanza rural en Gipuzkoa, 200-266.

${ }^{76}$ Archivo Universitario de Valladolid (AUV) Leg. 1350. En el informe elevado por el Rector de Valladolid a la Comisión de Cultura y Enseñanza, con fecha de 17 de noviembre de 1936, en el que incluían los nombres de los posibles integrantes de las Comisiones Depuradoras, se subraya que Josefina Olóriz tenía una «conducta inmejorable».

${ }^{77}$ La Comisión Depuradora de Gipuzkoa, fue creada por el Decreto n. ${ }^{\circ} 66$ de 8 de noviembre de 1936.
} 
conocimiento del euskara ${ }^{78}$ todas las propuestas en este sentido fueron frenadas por el Estado. No estaba en su ideario que se volviera a enseñar en euskara en las escuelas rurales vascas.

\section{ESTUDIO COMPARATIVO DE LOS DOS PROYECTOS}

La transformación social y económica que se estaba dando en las provincias vascas a principios del siglo $\mathrm{xx}$, y su realidad lingüística particular, priorizaba la modificación del su sistema educativo. Teniendo en cuanta la situación lingüística diferencial de estas provincias, sobre todo en las zonas rurales, las instituciones vascas eran conscientes de la necesidad de potenciar una educación que tuviera en cuenta la cultura y la lengua vasca. Partiendo de esta premisa, pero condicionados por un contexto y una realidad socio-política diferente, se diseñaron el Proyecto de Escuelas de Barriada de Bizkaia y el Proyecto de Escuelas Provinciales de Gipuzkoa. Estas experiencias, referentes en el desarrollo de la enseñanza rural, estuvieron condicionadas por la convulsa situación política de la época.

\section{La puesta en marcha de los dos proyectos}

En Bizkaia, la Corporación provincial nacionalista, surgida en las elecciones de 1917, fue pionera en la labor de diseñar un proyecto de escuela vasca que respondiera a las necesidades de la población escolar rural; así se diseñó el proyecto de Escuelas Barriada de Bizkaia. En 1919, después de ser aceptada la moción Gallano, se nombró a principios de 1920 una Ponencia ejecutiva conformada por Luis de Eleizalde, Eduardo de Landeta y los diputados Juan Gallano y Práxedes Aránsolo. Como resultado de su trabajo, en enero de 1921 ya funcionaban las dos primeras escuelas, la de Albiz de Mendata y Belendiz (Arratzu). ${ }^{79}$ El proyecto inicial, preconizaba escuela vasca con una enseñanza bilingüe, pero con la llegada de la Liga Monárquica a la Diputación, los cambios ideológicos fueron sustanciales. A pesar de todo, 15 años más tarde, aunque no con los planteamientos iniciales, eran 120 las escuelas de barriada que estaban en activo. ${ }^{80}$

\footnotetext{
${ }^{78}$ AGG (IT JD 874) Acta de la Diputación de Gipuzkoa, 15 de junio de 1940.

${ }^{79}$ Paliza, El proyecto de las escuelas de barriada, 59.

${ }^{80}$ Arrien, Educación y escuelas de Barriada en Bizkaia, 129-131.
} 
La Diputación Gipuzkoa, en 1930, y después de varios intentos que no tuvieron el éxito previsto, ${ }^{81}$ presentó el proyecto de Escuelas Rurales Provinciales. El objetivo principal de esta experiencia fue la erradicación del analfabetismo y la creación de nuevas escuelas en las zonas rurales. En estas escuelas se enseñaban las primeras letras en euskara, para después ejercitarse en lengua castellana. ${ }^{82}$ Las primeras escuelas se inauguraron en 1932, en los pueblos de: Zizurkil, Lezo, Legazpi, Oiartzun, Hernani y Beasain. ${ }^{83}$ En 1936 estaban en funcionamiento 21 escuelas.

En ambos modelos todo lo relativo a la creación, distribución, organización de estas escuelas competía a la Junta de Instrucción Pública de la Diputación. Hay que señalar que esta Junta delegaba, para más efectividad, la inspección general de las escuelas en la Junta local de cada pueblo (integrado por el alcalde, el cura párroco, el médico titular y tres padres de alumnos designados por los demás). ${ }^{84}$

La coyuntura política y social fue distinta en ambos proyectos y la dimensión del proyecto guipuzcoano fue de menor entidad. El presupuesto anual de las escuelas de Barriada de Bizkaia ascendía en 1930 a 1.037.750 pesetas; en Gipuzkoa, en los presupuestos de 1935, la cantidad adjudicada era de 269.926 pesetas. ${ }^{85}$

\section{Edificios escolares}

En Bizkaia, para la puesta en marcha de las escuelas de barriada, era condición necesaria que las barriadas o agrupaciones de caseríos tuvieran más de 10 vecinos, sus viviendas deberían de distar por lo menos

\footnotetext{
${ }^{81}$ AGG (JD IT 1874). En la sesión de 27 de diciembre de 1927, la Comisión de Enseñanza de la Diputación presentó un dictamen sobre Propuesta de normas para mejorar la instrucción primaria en las zonas rurales de la provincia, firmado por J. Rezola, J. Toledo y V. Ibarra. Con un presupuesto de unas 234.000 pesetas, este proyecto no se ejecutó, tal como se había previsto, por falta de financiación.

${ }^{82}$ Cándida Calvo Vicente, «La enseñanza en la zona rural guipuzcoana durante el primer franquismo», Vasconia, 27, (1998): 167. «En los lugares donde el vascuence sea el lenguaje usado —que es la totalidad en Guipúzcoa, puede decirse-, los niños recibirán la primera instrucción en euskera, aprendiendo así las primeras letras, para luego aprender el castellano».

${ }^{83}$ Joxe Garmendia, "Gipuzkoako landa auzoetan Udalek eta Aldundiak garatu dute hezkuntza-jarduera», Ikastaria, 16, (2008): 193-225.

${ }^{84}$ Zufía, Las Escuelas de Barriada. Apartado relativo a las Juntas locales de Inspección.

${ }^{85}$ Acta sesión de la Diputación de Gipuzkoa de 27 de diciembre de 1934.
} 
un kilómetro de la escuela más próxima y la barriada o ayuntamiento debería de proporcionar el local adecuado. ${ }^{86} \mathrm{El}$ edificio era, por lo general, de una sola planta, distribuida en pórtico, clase, cuarto de aseo, cuarto de material y cocina comedor. Lo único que sufría variación era el número de clases, condicionado por el número de alumnos. ${ }^{87}$ Por lo que respecta a su coste es difícil saber con certeza lo que costó cada una de las Escuelas de barriada, ya que muchos fueron construidos con las aportaciones voluntarias de los vecinos. Gregorio Arrien señala que, haciendo un cálculo medio, se puede valorar en unas 17.000 pesetas el coste de las escuelas sencillas y 24.000 pesetas el de las dobles. ${ }^{88}$

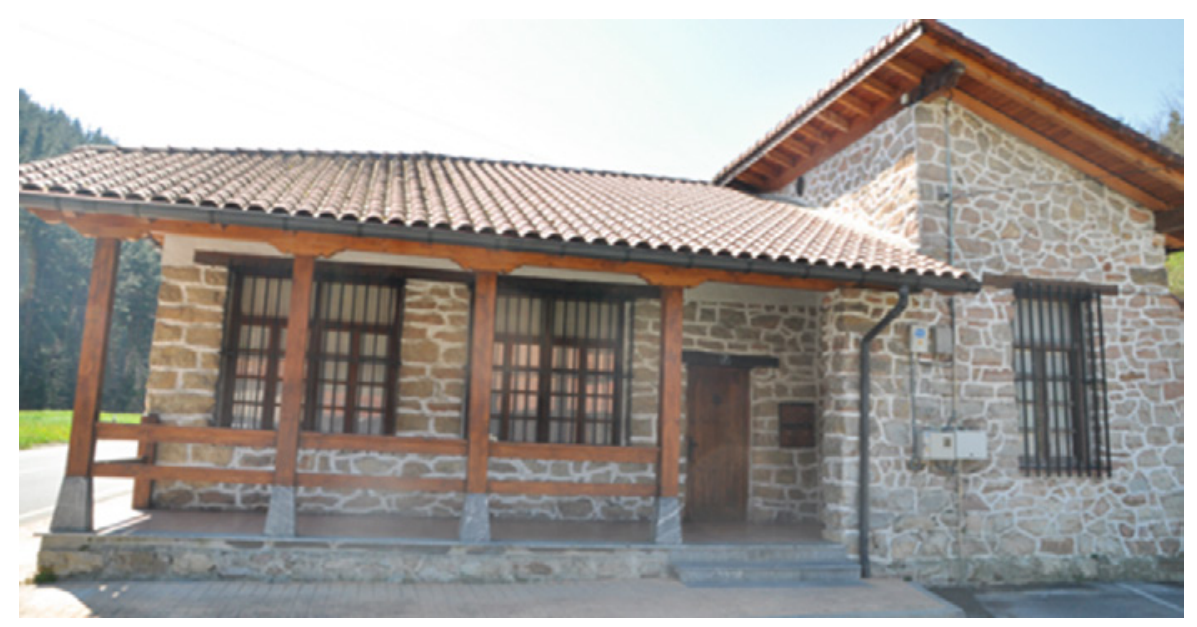

Foto 1. Escuela de barriada sencilla de Hiruzubieta en Ziortza-Bolibar. J. Garmendia

Por lo que respecta al número de escuelas, en 1925 se habían construido 77 escuelas, con 114 clases y otros tantos profesores. En 1930 se completaron las 100 escuelas (59 escuelas de una clase, 38 de dos clases —dobles_, 2 de tres clases y una de siete clases; en las escuelas dobles

\footnotetext{
${ }^{86}$ Zufía, Las Escuelas de Barriada en Vizcaya, 8. Pedro Zufía señala que a fin de 1920 había en Bizkaia 47 entidades de población con más de 100 edificios, 337 que tenían de 5 a 10 y 5.801 constituidas por menos de 5 edificios o caseríos aislados. El 35\% de la población estaba en núcleos menores de 10 casas.

${ }^{87}$ Zufía, Las Escuelas de Barriada. En el apartado correspondiente a Los locales escolares, describe estos edificios y su distribución.

${ }^{88}$ Arrien, Educación y escuelas, 139-158. Según circular y cuestionarios enviados a los ayuntamientos en 1920. En el caso de la escuela de la barriada de Albiz (Mendata) el coste del edificio escolar supuso unas 25.00 pesetas, incluido el terreno.
} 
había escuelas unitarias, 27, y escuelas graduadas, 11) que estaban previstas en el plan de la Diputación, alcanzando un censo escolar de más de 5.810 alumnos y 148 maestros. ${ }^{89}$ Durante la II República tuvo lugar una nueva ampliación de 25 escuelas, que se unieron a las ya existentes del plan anterior; al término del proceso constructivo en 1936 el número de alumnos ascendió a 6.321 y el de maestros a $162 .{ }^{90}$ La dirección técnica de las escuelas vizcaínas, que en algunos casos rehabilitó las escuelas existentes, estuvo en manos del arquitecto provincial Diego Basterra.

Las escuelas provinciales de Gipuzkoa, tuvieron como referencia el modelo de escuela de barriada, pero mejorado. En el proyecto guipuzcoano, al igual que en el de Bizkaia, también participaron los vecinos y las instituciones: la Caja de Ahorros Provincial aportó el 10\%, la Excma. Diputación el $25 \%$ y los respectivos Ayuntamientos y los vecinos el 65\% restante. En 1936, viendo el retraso en la creación de las escuelas, las condiciones iniciales fueron modificadas, y así a los ayuntamientos se les exigió que aportaran el terreno, con una superficie de 1.000 a 1.500 metros cuadrados y contribuyeran con el $25 \%$ de la liquidación de las obras. El resto iría a cargo de la Diputación. ${ }^{91}$

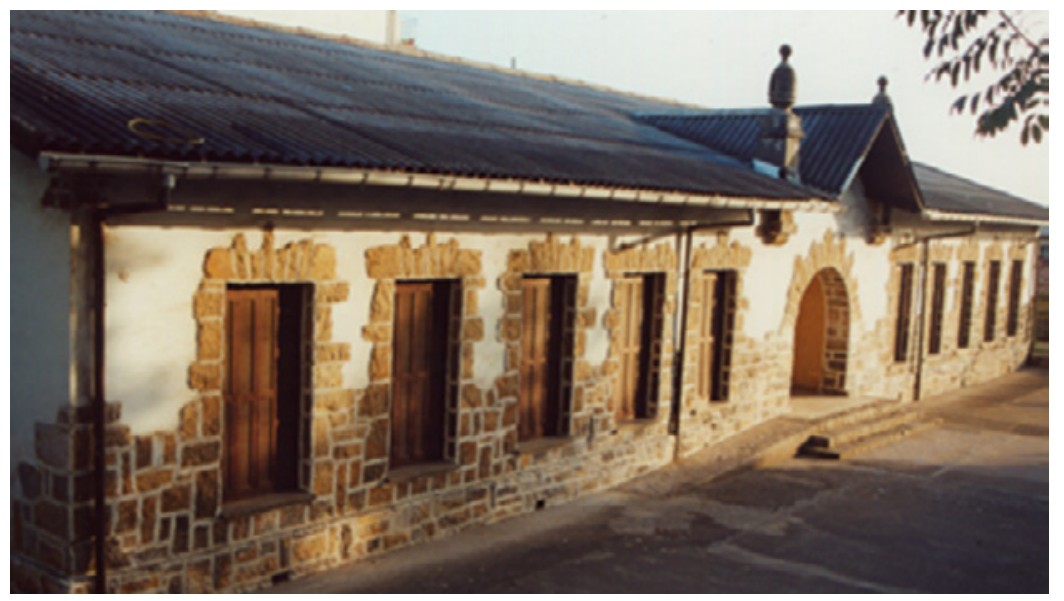

Foto 2. Escuela de barriada doble de Zizurkil. J. Garmendia

\footnotetext{
${ }^{89}$ Zufía, Las Escuelas de Barriada. Apartado relativo a la organización pedagógica.

${ }^{90}$ Arrien, Educación y escuelas 147.

${ }^{91}$ Boletín oficial de Guipúzcoa, de 1 de junio de 1936.
} 
El diseño de los nuevos edificios escolares guipuzcoanos fue obra del arquitecto de la Diputación Ramon Cortázar (1869-1944). ${ }^{92}$ En ambos proyectos las escuelas se ajustaban a las normas arquitectónicas impartidas por la Diputación, quien además de proporcionar el plano de la escuela, establecía también las condiciones de las edificaciones. Entre las particularidades de estas escuelas podemos señalar: calidad del espacio, higiene escolar, ventilación y luminosidad. ${ }^{93} \mathrm{El}$ material que se utilizaba era también en de buena calidad. En 1936, en Gipuzkoa, estaban en funcionamiento 21 escuelas y en proyecto otras 4 escuelas.

\section{Personal docente}

En Bizkaia, la provisión de plazas se realizó por concurso público, convocado con arreglo a una serie de bases entre las que primaban los valores personales, los conocimientos profesionales y el dominio de la lengua materna del alumno o alumna. En 1930, incluyendo los aspirantes, había en la categoría A, 3 maestros y 122 maestras; en la categoría B, 13 maestros y 41 maestras. Las oposiciones se repitieron con una regularidad casi anual. Antes del 1936 existieron 13 promociones diferentes, con una cifra de 177 maestros y maestras. Para tomar parte en la oposición se exigía el título oficial, capacidad física y sanitaria y la edad de 20 años para los maestros y 18 para las maestras.

Respecto a los sueldos que cobraban los maestros, hay que señalar que, aunque en los inicios la cantidad base era de 3.000 pesetas, el 1 de octubre de 1929 se fijó en 3.500 pesetas el sueldo anual de estos maestros. En 1931 el sueldo de los maestros ascendió a 4.000 pesetas. Esta anualidad iba aumentando, en quinquenios de 250 pesetas los dos primeros y de 500 los restantes, hasta llegar a las 5.000 pesetas anuales, cuyo máxi-

\footnotetext{
${ }^{92}$ Ramón Cortázar (1869-1944). Este arquitecto, fue el que diseñó la mayoría de estas escuelas y en la realización de las mismas siguió el denominado estilo neovasco, interpretación local del estilo denominado regionalismo. Joxe Garmendia, Hilario Murua e Iñaki Zabaleta, «De la oscuridad de las aulas de los caseríos a las exclusivas y peculiares escuelas rurales de la Diputación de Gipuzkoa (1931-36)», en Espacios y patrimonio histórico-educativo, ed. Paulí Dávila y Luis M. Naya (Donostia: Sociedad Española para el Estudio del Patrimonio Histórico-Educativo, 2016),402-405.

${ }^{93}$ Entrevista realizada a Marina Fernández por J. Garmendia en 26 de abril del año 2000. Esta maestra fue logró la primera plaza en la primera oposición de las escuelas provinciales de Gipuzkoa.
} 
mum no se pasaba. En el antiguo Reglamento se señalaba el derecho a jubilación, que podía ser voluntaria y forzosa. ${ }^{94}$

Entre las maestras de barriada podemos destacar la labor de Julene de Azpeitia (1888-1980) que fue una de las maestras más relevantes. ${ }^{95}$ Señalaremos, también, a Encarnación Velasco, Josefa Inés Aranzadi, Carmen Egia, Purificación Mandiola y Consuelo Gallastegi, entre otras, como destacadas maestras de barriada y miembros del movimiento femenino peneuvista de Emakume Abertzale Batza. ${ }^{96}$ La vocación de maestras, el conocimiento del euskara, sus ideales nacionalistas, junto a su condición femenina, les hacía candidatas perfectas para ocupar las plazas de maestras en las escuelas de barriada.

En Gipuzkoa los maestros y maestras fueron nombrados, previa oposición, por la Diputación. Debían poseer título oficial y conocer el vascuence (se les realizo un examen previo). El sueldo inicial era de 4.000 pesetas anuales, con cargo a los fondos provinciales, sin perjuicio de los aumentos graduales y derechos de jubilación que se estimasen conveniente fijar. Hubo dos pruebas de oposición que se desarrollaron en 1932 y en 1935. La mayoría de las maestras que lograron plaza habían sido alumnas de la Institución Teresiana. En este proyecto la mayoría de los docentes fueron mujeres, la excepción fue Juan Imaz que ejerció en la escuela de Zizurkil.

En Gipuzkoa en 1933, las maestras opositoras guipuzcoanas realizaron un cursillo formativo, tanto teórico como practico, que se desarrolló fundamentalmente en las escuelas de Fraisoro. En la parte práctica se trabajaron aspectos relacionados con la labor en el campo. ${ }^{97}$

\footnotetext{
${ }^{94}$ Arrien, Educación y escuelas, 140

${ }^{95}$ Nacida en Zumaia en 1888, en 1911, se presentó a las oposiciones que se realizaron en Valladolid y quedó en primer lugar; a continuación, desarrollo su labor pedagógica en las ikastolas de la capital vizcaína. Entre otros, presentó el trabajo Irakurri maitte al concurso pedagógico que organizó la Diputación de Vizcaya en 1925 y se llevó el primer premio. Julene estuvo integrada en la Agrupación de Maestros Vascos de Bilbao, asociación que pretendía influir en la organización y marcha de las escuelas de barriada, sin grandes resultados. Más información sobre esta maestra en Laura Mintegi, Julene Azpeitia. Bidegileak (Vitoria-Gasteiz: Eusko Jaurlaritza-Kultura saila, 1987).

${ }^{96}$ Movimiento de las mujeres del Partido Nacionalista Vasco, que tuvo una gran relevancia en los años de la postguerra. Itziar Recalde, Escuela y sociedad en Euskadi. La enseñanza primaria (Salamanca, Universidad de Salamanca, 2001), 305. Policarpo de Larrañaga, Emakume Abertzale Batza (Donostia, Auñamendi, 1978).

${ }^{97}$ Acta de la Diputación de Gipuzkoa. 27 de julio de 1933.
} 


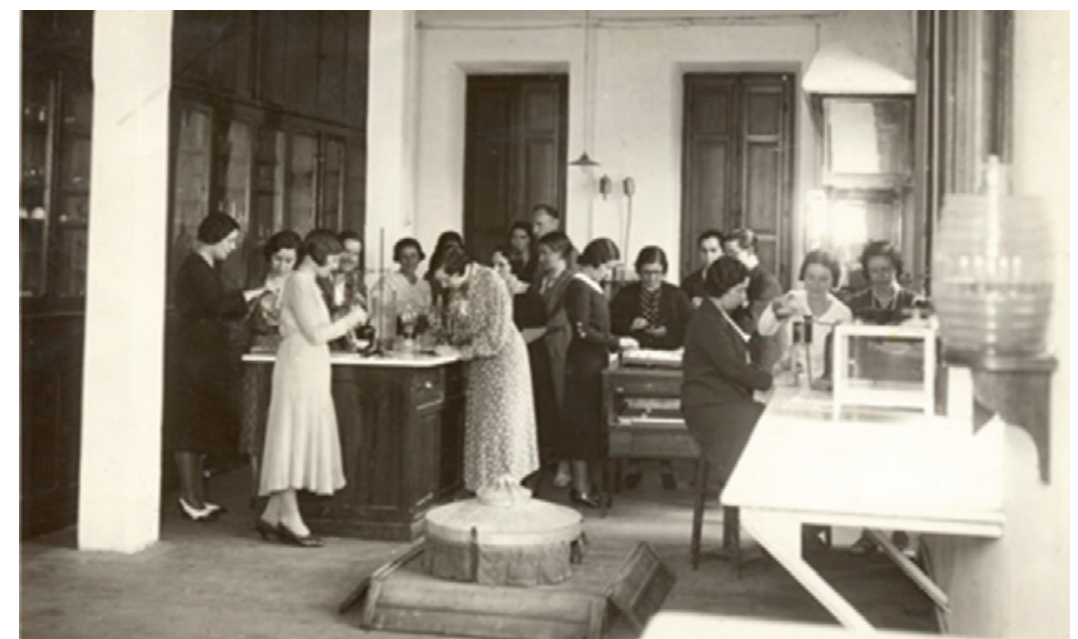

Foto 3. Formación de maestras provinciales de Gipuzkoa. Marina Fernández

\section{Inspección}

Por lo que respecta al cargo de Inspector de las escuelas de Bizkaia, sus atribuciones consistían en: asesorar a la Junta, la orientación del personal docente y la inspección de los establecimientos. En esta labor de inspección hay que destacar a Luis de Eleizalde, inspector entre los años 1919 a 1923, que fue elegido en oposición entre otros 14 aspirantes, ${ }^{98}$ y a Pedro de Zufía, que le sucedió entre los años 1924 a 1937.

En Gipuzkoa, la Inspección estuvo a cargo de Josefina Olóriz, maestra teresiana conocedora de las innovaciones pedagógicas del momento y defensora de los principios religiosos que promovía el padre Poveda, por lo que disentirá de muchas de las ideas de la República. Apreciada por unos, fue muy criticada por las fuerzas republicanas y nacionalistas. A pesar de que no dominaba el euskara, fue elegida para dirigir a unas maestras que tuvieron que superar una prueba de euskara para lograr su plaza.

\section{Organización escolar y pedagógica}

En el proyecto de las escuelas de barriada de Bizkaia las escuelas estaban clasificadas en mixtas, unitarias y especiales o graduadas; la mayor

\footnotetext{
${ }^{98}$ Arrien, Educación y escuelas, 162.
} 
parte de estas últimas eran de dos grados. ${ }^{99}$ En el Reglamento de 1926 se señalaba que la edad de los alumnos estaba comprendida entre los 5 y los 14 años, a razón de dos años en cada uno de los siguientes niveles: preparatorio, elemental, medio y superior. ${ }^{100} \mathrm{El}$ censo escolar nunca debía superar los 48 alumnos por aula.

Entre las particularidades pedagógicas de estas escuelas señalaremos las siguientes: la enseñanza era absolutamente gratuita y pública; teniendo en cuenta las distancias y las condiciones de los caminos a recorrer, la Diputación estableció todas las escuelas una Cantina, que era un departamento con cocina, fregadera y comedor; también se crearon las Bibliotecas escolares y se otorgaron becas de 500 a 2.000 pesetas anuales, diez cada año. Entre las actividades complementarias cabe mencionar la mutualidad escolar, las clases de adultos, las excursiones escolares con fines educativos, los museos y exposiciones. Por lo que respecta a la pedagogía, hay que señalar que se rechazaban categóricamente las lecciones de memoria y el uso excesivo de los libros de texto, recomendándose en su lugar la conversación viva, la realización de ejercicios prácticos y las excursiones. Las disciplinas que presentaba el programa eran las siguientes: Instrucción moral y religiosa (catecismo y sagradas escrituras), Lenguaje (lectura, escritura y gramática), Aritmética y geometría (calculo, problemas, sistema métrico decimal,..), Ciencias físico-naturales (en íntima relación con la agricultura), Geografía e Historia (estudio de la barriada, el pueblo, la provincia,...), Fisiología e Higiene (higiene personal, doméstica y social), Enseñanzas prácticas (dibujo, labores agrícolas, labores femeninas), Trabajos manuales (trabajos en madera, hierro, confección de redes,...), Cantos, Gimnasia. ${ }^{101}$

La lengua en la que se impartiría la docencia fue un elemento clave en la organización de estas escuelas. Las escuelas de barriada fueron agrupadas en dos categorías A y B. En la primera la enseñanza se impartía en euskara, siendo materia obligatoria el castellano, mientras que en las

\footnotetext{
${ }^{99}$ Arrien, Educación y escuelas, 160.

${ }^{100}$ Zufía, Las Escuelas de Barriada. Tenemos que añadir que en el primer Reglamento (ADV Legajo 2731), el periodo de asistencia estaba comprendido entre seis y los trece años divididos en tres partes: Curso elemental (6 a 8 años), Curso medio (de 8 a 10 años) y Curso superior (de 10 a 13). Las asignaturas se organizaban teniendo en cuenta las características de cada curso, categoría y pueblo. ${ }^{101}$ Zufía, Las Escuelas de Barriada. Apartados relativos a la organización pedagógica y el programa escolar.
} 
segundas el sistema era inverso. ${ }^{102} \mathrm{Si}$ en un primer momento, con Eleizalde de inspector, el objetivo de estas escuelas estuvo dirigido a crear una escuela vasca y bilingüe, con la llegada de la Liga Monárquica y la dictadura de Primo de Rivera, hubo un drástico cambio en los planteamientos. Los monárquicos se adaptaron bien al nuevo régimen, pero para los socialistas y nacionalistas no fue nada fácil. En 1925, la Real orden de 16 de octubre aprobó la apertura de las escuelas pero ponía una serie de condiciones: ${ }^{103}$

3. La enseñanza se dará siempre en el idioma castellano.

4. Estas escuelas serán visitadas, al igual que las nacionales, por la Inspección de Primera Enseñanza del Estado, que deberá dar inmediata cuenta de cualquier anomalía.

Con estos cambios radicales, lejos quedaba el primer Reglamento de 1921 que señalaba que en las escuelas de categoría A se daría la enseñanza en lengua vasca.

Por lo que respecta a las escuelas guipuzcoanas, hay que señalar que fue deseo de la Diputación de Gipuzkoa que las escuelas de barriada de Bizkaia fueran un referente a la hora de organizar su proyecto. Así, la inspectora a las escuelas provinciales de Gipuzkoa, Josefina Olóriz, visitó en 1930 las escuelas de barriada a fin de conocer el desarrollo de las mismas. Las escuelas de Gipuzkoa se diseñaron conforme al pensamiento pedagógico de esta inspectora y en la línea de las escuelas de Bizkaia. Así, se rechazaban las lecciones de memoria y se insistía en que la finalidad era fomentar en el niño el amor a la verdad y la justicia y despertar el gusto por las bellezas artísticas y de la naturaleza. Similares objetivos pueden leerse en otros modelos de programas publicados en el Estado español. ${ }^{104}$ Se reivindicaba una metodología práctica y utilitaria, en oposición a los sistemas de preguntas y respuestas, de definiciones, etc. La corta duración del proyecto guipuzcoano imposibilitó que se pudiera realizar un estudio de la enseñanza que se había comenzado a impartir. Tampoco la

\footnotetext{
102 Diputación de Bizkaia, Escuelas de Barriada de Vizcaya. Moción Gallano: estatutos, reglamento, plan de cuadros y enseñanza (Bilbao: Imp. Provincial, 1921).

${ }^{103}$ R. O. del 16-X-1925 (BOPV de 31-XI-1925, folio 1.057, n. ${ }^{\circ}$ 247).

${ }^{104}$ AADG, Expediente 36-18. Reunión de la Comisión de Enseñanza de 1 de abril de 1931.
} 
Inspección tuvo tiempo para elaborar un informe sobre el desarrollo de las mismas.

Hay que señalar también que, a partir de 1931, durante el mandato de la izquierda, en ambos proyectos se prohibió la iconografía religiosa y el impartir la enseñanza religiosa en las escuelas. A pesar de la prohibición, de lo que no hay duda es que, en la mayoría de los casos, las maestras de estas escuelas rurales y la inspección no se oponían a la enseñanza de la religión. ${ }^{105}$

\section{Material escolar}

En ambos proyectos, la mayor parte del material escolar era proporcionado gratuitamente por la Diputación. A estas escuelas se les dotó del material fijo y móvil más moderno. En las escuelas de barriada de Bizkaia en los primeros años se utilizaron textos en euskara. Los primeros libros fueron escogidos mediante concurso público; así podemos destacar los siguientes libros: Euzko erixen edesti laburra, de Eugenio Urroz y Gabriel Manterola; Ume euzkeldunen laguna (lenengo eta bigárren edazkija) de Joseba Altuna, y Azijerea ta Ozazunbidea, de C. de Jemein ${ }^{106}$. Eleizalde también elaboró el texto de Aritmética elemental Euzkal-Zenbakiztia. Se publicaron también silabarios como Euzkeraz Irakuŕteko ta lenengo irakurkizunak y también un catecismo en euskera, el Kristiñau Ikasbidea, de primer grado. Estos textos, en la mayoría de las veces, se imprimían en euskera vizcaíno y en la ortografía que seguía las pautas señalas por Sabino Arana-Goiri. Entre los libros publicados en 1920 por la Sociedad de Estudios Vascos, sobre todo para estas escuelas, señalaremos el Umearen laguna, irakurtzen ikasteko biderik érezena, de Lopez de Mendizabal y Txomin Irakasle y Sabin Euskalduna, de B. Estornés. Además de estos libros también se publicaron otros textos sobre Aritmética, Ciencias, Geografía e Historia. Pero, sin duda, donde mejor se recoge el universo nacionalista es en el libro Xabiertxo, Umeei euskeraz irakurtzen

\footnotetext{
${ }^{105}$ AGA. Sección Educación, Leg. 192-58, del 2.X.1940. En el informe de Depuración de la maestra a Delfina Pérez, acusada de simpatizante de izquierdas, se señalaba: «Los niños no dejaban de dar su clase de catecismo, cuando en la escuela estaba vedada su enseñanza» En el informe de M. L. Olano de San Blas-Tolosa, acusada de ser simpatizante de un partido de izquierda se indicaba: «Esta maestra, en pleno gobierno del Frente Popular daba la clase Doctrina Cristiana, fuera de las horas de clase».

${ }^{106}$ Arrien, Educación y escuelas, 172-173.
} 
erakusteko idaztia (Xabiertxo, escrito para enseñar a leer a los niños en euskara). ${ }^{107}$ La falta de un lenguaje unificado retrasó de forma considerable la aparición de textos traducidos de otros idiomas, como el castellano o el francés.

Con la llegada de los monárquicos a la Diputación de Bizkaia, se abandonó la educación bilingüe de los inicios, permitiéndose en adelante el uso del euskera sólo como vehículo o medio de enseñanza, o para el uso del catecismo. Al estar prohibidos los libros en euskara, serán los libros editados en castellano los más utilizados.

En Gipuzkoa, al igual que había sucedido en Bizkaia, la creación de las nuevas escuelas rurales trajo consigo la necesidad de reutilizar los textos que se habían creado para las escuelas de barriada y de crear nuevas publicaciones y textos en euskara. Entre los libros en euskera más utilizados podemos señalar los libros antes citados: Xabiertxo, umiei euskeraz irakurtzen erakusteko idatzia (1925) y el silabario de Ixaka Lopez de Mendizabal, Martín Txilibitu: Umearen laguna (1931). También se utilizaron los libros de Fermín Iturrioz Txomin Irakasle (1931), ${ }^{108}$ Lutelesti edo Jeographia —Geografía General y regional bilingüe (1932)—, ${ }^{109}$ Sabin euskalduna (1931) de Bernardo Estornés Lasa e ilustrado por Txiki ${ }^{110}$ y Nekazaritzako irakurraldiak - Lecturas agropecuarias (1933). ${ }^{111}$ A falta de libros y material apropiado, muchas escuelas, los temas se solían impartir con los apuntes que confeccionaba la maestra.

Entre los libros en castellano utilizados en estas escuelas señalaremos: Lecturas Geográficas, Historia Universal, Geometría, Lecciones de Cosas, Geografía Física y Astronomía y Aritmética de las editoriales Palau Vera y Seix Barral. Respecto a material móvil (mesas, bancos y material pedagógico), la Casa Espasa-Calpe fue la que proveía a las escuelas rurales provinciales. ${ }^{112}$

\footnotetext{
${ }^{107}$ El libro Xabiertxo de I. Lopez de Mendizabal, premiado por la Sociedad «Euskalerriaren-Alde. Ilustrado por el dibujante «Txiki», aparecerá en 1925, en plena Dictadura.

${ }^{108}$ Petición de ayuda para sus publicaciones de 30 septiembre de 1931.

${ }^{109}$ Acta de la sesión Diputación de Gipuzkoa de 16 de junio de 1932.

${ }^{110}$ AGG (JD IT 1457/2001). Petición de ayuda para sus publicaciones de 6 de marzo de 1932.

${ }^{111}$ Acta de la Diputación de Gipuzkoa, 28 de julio 1932.

112 AGG (JD IT 1457/2002).
} 


\section{Depuración y supresión de las escuelas}

En las provincias vascas, desde un primer momento, los sublevados trataron de hacerse con el control del sistema educativo; esto fue sólo el preludio de la depuración ideológica que inmediatamente se puso en marcha. ${ }^{113}$ Una vez tomado el poder, la atención de las nuevas autoridades golpistas se dirigió a la depuración del personal docente. A este respecto, deben de destacarse dos disposiciones importantes: la Orden de 8 de noviembre y la de 7 de diciembre de 1936. ${ }^{114}$

En Bizkaia fueron definitivamente destituidos de su puesto en la Diputación 463 trabajadores y, entre estos, 35 maestros y maestras de barriada. Estos fueron destituidos por considerar que su actuación se hallaba comprendida dentro de los castigos y puntos tipificados. ${ }^{115}$ Así, se sustituyó a los maestros y maestras que habían tenido relación con el Frente Popular o habían apoyado sus métodos escolares y, sobre todo, a los maestros de influencias nacionalistas o los que habían destacado en la defensa de la lengua vasca. ${ }^{116}$

En Gipuzkoa, la represión llegó también a estas escuelas provinciales ${ }^{117}$ y a las escuelitas. ${ }^{118}$ Un considerable número de docentes fueron suspendidos o apartados de sus cargos, la mayoría de ellos por su afinidad política. Así muchos maestros y maestras fueron depurados y las escuelas cerradas. La guerra trajo también la desaparición del cuerpo de mikeletes y, con ello, de las escuelas que estaban a su cargo.

\footnotetext{
${ }^{113}$ Hilario Murua, La enseñanza primaria y el magisterio en Gipuzkoa, 1936-1975 (Bilbao: EHU-UPV, 2007), 44-61.

${ }^{114}$ Maitane Ostolaza, El garrote de la depuración. Maestros vascos en la guerra civil y el primer franquismo, 1936-1945, (Donostia: Ibaeta-Pedagogía, 1996), 101-170.

${ }^{115}$ Arrien, Educación y escuelas, 203. Arrien señala que en Bizkaia fueron repuestos 92 maestros, 35 fueron destituidos por considerar que su actuación se hallaba comprendida dentro de los castigos y puntos tipificados.

${ }^{116}$ Arrien, Educación y escuelas, 631-635.

${ }^{117}$ AUV. Legajo 2718, con registro de entrada 11.III.1937. Dos maestras provinciales tenían informes desfavorables. M. ${ }^{a}$ Luisa Olano de Tolosa y Delfina Perez de Oyarzun.

${ }^{118}$ Acta de la Diputación de Gipuzkoa de 11 de febrero de 1937 e información directa recogida por el autor, Joxe Garmendia, en entrevistas personales. En 1937 el número de maestras y maestros de escuelitas depuradas ascendía al menos a 20 escuelas.
} 
En abril de 1938 las escuelas provinciales fueron declaradas escuelas nacionales. ${ }^{119}$ La Orden de 24 de noviembre de 1938 aprobó las normas para reglamentar el traspaso de los centros citados al Estado, dependiendo en adelante del Ministerio de Educación Nacional. Suprimidos los Conciertos Económicos, la Diputación, a partir del presupuesto de 1938, suspendió también las partidas destinadas a la mayoría de las escuelitas rurales particulares. En situación de pobreza generalizada, las escuelitas que no fueron cerradas o depuradas tuvieron que subsistir en unas condiciones deficientes y precarias.

\section{CONCLUSIÓN}

El primer tercio del siglo xx fue un periodo de profundos cambios sociales y políticos en Gipuzkoa y en Bizkaia. Por lo que respecta al proceso educativo, el reto del proceso modernizador suponía sustituir un modelo de escuela que abandonado a su suerte: con un magisterio no cualificado, con material y edificios deficientes, y con un gran absentismo escolar. El objetivo era el crear unas escuelas modernas y bajar las tasas de analfabetismo, sobre todo en el ámbito rural.

Hay que destacar que, con la puesta en marcha de los proyectos de las escuelas de barriada y las escuelas provinciales, se trató de corregir el desajuste educativo de las zonas rurales, con unos edificios peculiares y con una organización envidiable. El nuevo modelo de escuela rural, con escuelas de nueva edificación, bien equipadas — con frontón, cantina, calefacción, libros-, con un personal formado, titulado y bilingüe, con una enseñanza gratuita, fue un lujo que no se había conocido hasta entonces en los barrios rurales. Estas escuelas contaron con una adhesión popular muy elevada.

El desarrollo de estas experiencias educativas, que tenían en cuenta las particularidades de la sociedad rural vasca, fue un hito muy importante en la escolarización y alfabetización de los niños y niñas del mundo rural, sobre todo en Bizkaia. En la realización de este proyecto, las diversas diputaciones ejercieron una labor semejante a la que ejercía el Estado en las escuelas nacionales: participando y dirigiendo la construcción de edificios; aportando los materiales y el mobiliario escolar; haciéndose

\footnotetext{
${ }^{119}$ Itziar Rekalde, Escuela, educación e infancia durante la guerra civil en Euskadi, 305.
} 
cargo de las retribuciones del profesorado, y marcando las normas para su elección, remuneración, orientación y control.

Si se hubiesen desarrollado en su totalidad las distintas experiencias, tal como se diseñaron en un principio, es indudable que hubieran tenido repercusiones muy importantes en la formación de los campesinos vascos. Comparando ambas experiencias, tenemos que señalar que la coyuntura política y social fue distinta en ambas y que, a causa sobre todo de la escasez de recursos, la dimensión del proyecto guipuzcoano fue de menor entidad.

Al finalizar la guerra civil en el País Vasco, en 1938, las escuelas provinciales de Bizkaia y de Gipuzkoa fueron declaradas escuelas nacionales y las maestras y maestros que no se habían alineado con las fuerzas franquistas, sufrieron una dura persecución. Es preciso señalar que muchas de las maestras de las escuelas de barriada eran militantes y simpatizantes nacionalistas. En Gipuzkoa, la represión también llegó a las maestras de las escuelas rurales y escuelitas que se habían identificado con los movimientos nacionalistas.

En 1938, en Gipuzkoa el Estado se hizo cargo de las 21 escuelas que estaban abiertas, pero no procedió a la creación del resto de las 79 escuelas que se habían previsto. La construcción de cien escuelas rurales no fue más que una aspiración irrealizada. En consecuencia, el problema de la enseñanza rural siguió sin resolverse.

Para finalizar debemos de señalar que, a pesar de todas sus carencias y vicisitudes, no se puede negar la mayúscula aportación de estas escuelas rurales en la renovación educativa, la alfabetización y la enseñanza en euskera en los barrios rurales. Aunque fuere por poco tiempo, los padres $\mathrm{y}$ alumnos de estas zonas rurales pudieron disfrutar de un modelo educativo innovador que hasta ese momento era impensable.

A día de hoy, en la segunda década del siglo XXI y con una realidad socio-política, lingüística y de servicios muy diferente, podemos afirmar que la alfabetización y la enseñanza en euskara, incluso en las zonas rurales, es una asignatura superada. Mirando al futuro, los nuevos retos de la enseñanza rural van en la línea de superar el proceso de concentración de las escuelas rurales que se dio sobre todo a finales del siglo xx y revitalizar la enseñanza rural abriendo nuevas escuelas con nuevos servicios. 
Así, en el País Vasco, en los pequeños núcleos de población de apenas varios centenares de habitantes o en los barrios alejados del centro educativo de su municipio, los profesionales, los padres de los alumnos y el Departamento de Educación están potenciando un modelo de escuela pública en euskara, el modelo de Escuelas Pequeñas, Eskola Txikiak. En estas escuelas creadas a partir de 1987 — con grupos de diferentes edades y con menos de seis aulas, incluyendo la enseñanza preescolar y primaria- los niños y niñas crecen en una escuela ligada al pequeño pueblo o barrio rural, manteniendo la forma de vida, las costumbres y la cultura del lugar. Es evidente que estas escuelas, en muchos casos son elementos necesarios para hacer frente al despoblamiento, e imprescindibles si queremos lograr un futuro sostenible en los pequeños pueblos rurales. Estas nuevas escuelitas -26 en Gipuzkoa, 14 en Bizkaia y 10 en Álava- son el futuro de la enseñanza rural y, aunque representan una cantidad poco relevante en el sistema educativo vasco, tienen una enorme importancia cualitativa.

\section{Nota sobre los autores}

JoXe GaRMENDIA LARRAÑAGA es en la actualidad profesor agregado en la UPV-EHU, impartiendo docencia en la Facultad de Educación, Filosofía y Antropología de Donostia-San Sebastián Diplomado en Magisterio (1981) y licenciado en Pedagogía (1993) y Doctor en Filosofía y Ciencias de la Educación por la UPV-EHU (2003). Obtuvo el doctorado presentando la tesis Enseñanza rural en Gipuzkoa. La labor de la Diputación y los ayuntamientos 1900-1950 en marzo de 2003. En su trayectoria académica y profesional, ha participado en numerosos proyectos de investigación relacionados con la historia de la educación. Asimismo, es autor de los varios libros y artículos relacionados con la educación en el País Vasco, entre los que citaremos: Azkoitiko bailaretako eskolak 1824-1977, "Las escuelas rurales de Gipuzkoa. El proyecto de las escuelas provinciales 1930-1950», «Movimiento popular y resistencia frente al sistema educativo franquista: las Ikastolas», «Ordizia Hiri Hezitzailea»; etc. Es miembro del Grupo Consolidado de Investigación Garaian, dirigido por el catedrático D. Paulí Dávila, grupo que forma parte de la Unidad de Formación e Investigación «Educación, Cultura y Sociedad» de la UPV-EHU. Ha cola- 
borado, también, en distintos proyectos internacionales de investigación sobre inmigrantes bajo la dirección del catedrático D. Felix Etxeberria.

IÑAKi ZaBALETA ImAZ es Licenciado en Filosofía y Ciencias de la Educación y Doctor en Pedagogía es Profesor Titular de Universidad en la Universidad del País Vasco / Euskal Herriko Unibertsitatea y miembro del Departamento de Teoría e Historia de la Educación de la misma. Desarrolla su tarea docente en la Facultad de Educación, Filosofía y Antropología de Donostia-San Sebastián Respecto a tareas de gestión, entre otras funciones, ha desempeñado el cargo de Director del Departamento de Teoría e Historia de la Educación, ha sido Decano de la Facultad de Filosofía y Ciencias de la Educación y miembro del Consejo de Gobierno de la Universidad del País Vasco /Euskal Herriko Unibertsitatea.

Por otra parte, ha participado en diferentes proyectos de investigación. También es miembro del grupo consolidado de investigación Garaian (Grupo de Investigación en Estudios Históricos y Comparados en Educación). Las líneas fundamentales de investigación sobre las que ha desarrollado su trabajo son las siguientes: política educativa, nacionalismo vasco, infancia, escuela vasca, alfabetización, formación profesional, profesorado, etc. Asimismo, ha publicado diferentes artículos y libros, algunos de los cuales se citan a continuación: Euskal nazionalismoa eta hezkuntza (1895-1923); Nazioa eta hezkuntza-sistema espainiarraren sorreran, etc. Por otra parte, también ha publicado diferentes trabajos en colaboración con otros investigadores: "The construction of the Basque Fatherland: religion, nationalism and education (1895-1931)», etc.

Hilario Murua Cartón es profesor adjunto en la UPV/EHU; impartiendo docencia en la Facultad de Educación, Filosofía y Antropología de Donostia-San Sebastián. Diplomado en Magisterio (1992), licenciado en Pedagogía (2000) y Doctor en Pedagogía por la UPV/EHU (2006), obtuvo el doctorado con la tesis denominada La Enseñanza Primaria y el Magisterio en Gipuzkoa, 1936-1975. En su trayectoria profesional ha participado en diferentes proyectos de investigación relacionados con la Teoría e Historia de la Educación. Asimismo, es autor de varios libros y artículos relacionados con la Teoría e Historia de la Educación: La Enseñanza Primaria y el Magisterio en Gipuzkoa, 1936-1975, 100 años de La Salle en Gipuzkoa, La Formación Profesional en Euskal Herria: evolución y agentes promotores durante el franquismo, etc. Es miembro del Grupo Consolidado de Inves- 
tigación Garaian, dirigido por el catedrático D. Paulí Dávila, grupo que forma parte de la Unidad de Formación e Investigación «Educación, Cultura y Sociedad» de la UPV-EHU. Ha colaborado, también, en distintos proyectos internacionales de investigación sobre inmigrantes bajo la dirección del catedrático D. Felix Etxeberria.

\section{REFERENCIAS}

AizPuru MuruA, Mikel. «El agrarismo guipuzcoano y el nacionalismo vasco (1920-1933)». Historia Agraria 70 (2016): 167-194.

Álvarez Junco, José. Mater Dolorosa. La idea de España en el siglo XIX. Madrid: Taurus, 2001.

Ariztimuño, Jose. Obras Completas 2. La democracia en Euzkadi. Lucha de idiomas en Euzkadi y en Europa. Donostia: Erein, 1986.

Arpal Jesús, Begoña Asua y Paulí Davila. Sociedad y Educación en el País Vasco. San Sebastian: Txertoa, 1982.

ARRIEN, Gregorio. Educación y escuelas de Barriada en Bizkaia. Escuela y Autonomía 1898-1936. Bilbo: Bizkaiko Foru Aldundia, 1987.

Bilbao, Begoña, Gurutze EzKurdia y Karmele Perez. Euskal Curriculuma ala Euskal Dimentsioa Curriculumean?. Donostia: Utriusque Vasconiae, 2004.

CALvo Vicente, Cándida. "La enseñanza en la zona rural guipuzcoana en el primer franquismo». Vasconia 27 (1998): 166.

CASTELLS, Luis. Modernización y dinámica política en la sociedad Guipuzcoana de la Restauración (1876-1915). Madrid: Siglo XXI, 1987.

Costa, Anton. "Escolas en contornas rurais en Galicia: horizonte difícil/horizonte posible». Innovación Educativa 24 (2014): 33-153.

DÁvila, Paulí. La política educativa y la enseñanza pública en el País Vasco (18601930). San Sebastián: Ibaeta Pedagogía, 1995.

- Lengua escuela y cultura. El proceso de alfabetización en Euskal Herria, siglos XIX Y XX. Donostia: EHU, 1998.

- «Curriculum vasco, agentes sociales y libros de texto en euskara hasta 1937». Euskonews 265 (2008): 12-19. Consultado el 11 de enero 2017. http://www. euskonews.com/0465zbk/gaia46504es.html.

— «Euskal Herria tiene forma de corazón». Historia de la educación 27 (2008): 215-243.

DÁvila, Paulí y Ana EIzAgIRRE. «Alfabetización y euskaldunización en Euskal Herria». In Leer y Escribir en España, doscientos años de alfabetización, edited by Agustín Escolano,187-211. Madrid: Fundación Sánchez Ruiperez, 1992.

Delgado, Ander. «¿Amantes de Dios de la patria y de la familia?». Educatio siglo XXI 34 (3), (2016): 47-64. 
Diputación de Vizcaya. Escuelas de Barriada de Vizcaya. Moción Gallano: estatutos, reglamento, plan de cuadros y enseñanza. Bilbao: Imp. Provincial, 1921.

Estornés, Idoia. La Sociedad de estudios vascos. La aportación de Eusko Ikaskuntza a la cultura vasca (1918-1936.). San Sebastián: Eusko Ikaskuntza, 1983.

EzKURDIA ARTEAGA, Gurutze. «Euskal Herria gaiaren garapena XX. eta XXI. mendeetako testuliburuetan». Ikastaria 16 (2008): 109-142.

GABRIEL, Narciso de. Escolantes e Escolas de Ferrado. Vigo: Xerais, 2001.

GARMENDIA, Joxe. «Mikelete postuetako eskolak». Hik Hasi 62 (2001): 41-43.

- La enseñanza rural en Gipuzkoa. La labor de la Diputación y los Ayuntamientos, 1900-1950. Bilbao: Universidad del País Vasco, 2004.

- «Gipuzkoako landa auzoetan Udalek eta Aldundiak garatu dute hezkuntza-jarduera». Ikastaria 16 (2008): 193-225.

Garmendia, Joxe, Hilario Murua e Iñaki ZabaletA. «De la oscuridad de las aulas de los caseríos a las exclusivas y peculiares escuelas rurales de la Diputación de Gipuzkoa (1931-36)». In Espacios y patrimonio histórico-educativo, edited by Paulí Dávila y Luis M. Naya, 402-405.Donostia: Sociedad Española para el Estudio del Patrimonio Histórico-Educativo, 2016.

GELLnER, Ernest. Naciones y nacionalismos. Madrid: Alianza, 1988.

HERnÁNDEz DíAz, José María. «La escuela rural en la España del siglo XX». Revista de Educación, Número Extraordinario (2000): 113-136.

Hobsbawm, Eric. Naciones y nacionalismo desde 1780. Barcelona: Edición Crítica, 1991.

IzTUeTA, Paulo. «Las contradicciones de la cultura vasca: Teoría y praxis». In Lengua, escuela y cultura. El proceso de alfabetización en Euskal Herria, siglos XIX y XX, edited by Paulí Dávila, 349-50. Bilbao: Universidad del País Vasco, 1995.

Larrañaga, Policarpo de. Emakume Abertzale Batza. Donostia: Auñamendi, 1978. LARRONDE, Jean-Claude. El nacionalismo vasco: su origen y su ideología en la obra de Sabino Arana-Goiri. San Sebastián: Ediciones Vascas, 1977.

Letamendia, Francisco. El hilo invisible. Bilbao: EHU-UPV, 2013.

LÓPEZ, Ramón. «La construcción y creación de escuelas en la España del primer tercio del siglo XX». Historia de la educación 16 (1997): 65-90.

LóPEz Adán, Emilio. El Nacionalismo Vasco (1876-1936). San Sebastián: Txertoa, 1977.

MARtínez Domínguez, Blanca. «La formación de capital humano en Galicia». Revista Galega de Economía 12 (1), (2003): 1-22.

Mintegi, Laura. Julene Azpeitia. Bidegileak. Vitoria-Gasteiz: Eusko Jaurlaritza-Kultura saila, 1987. 
Murua, Hilario. La enseñanza primaria y el magisterio en Gipuzkoa, 1936-1975. Bilbao: EHU-UPV, 2007.

Núñez SEIXAs, Xosé Manoel. Los nacionalismos en la España contemporánea siglos XIX y XX. Barcelona: Hipòtesi, 1999.

OstolazA, Maitane. El garrote de la depuración. Maestros vascos en la guerra civil y el primer franquismo, 1936-1945. Donostia: Ibaeta-Pedagogía, 1996.

PalizA, Maite. El proyecto de las escuelas de barriada su desarrollo (1919-1938) Una epopeya de la historia de la educación en Bizmaría. Bilbao: Gobierno Vasco, 2015.

RECALDE, Itziar. Escuela y sociedad en Euskadi. La enseñanza primaria. Salamanca: Universidad de Salamanca, 2001.

RIQUER, Borja de. Escolta Espanya. La cuestión catalana en la España liberal. Madrid: Marcial Pons, 2002.

RosiQue, Francisca. Historia de la Institución Teresiana (1911-1936). Madrid: Sílex. 1914.

Rubio PoBes, Coro. La identidad vasca en el siglo XIX. Discurso y agentes sociales. Madrid: Biblioteca Nueva, 2003.

TiveY, Leonard. El Estado Nación. Barcelona: Nova Grafic, 1987.

ZABALETA, Iñaki. «La enseñanza en los orígenes del nacionalismo vasco: la cuestión religiosa». In Las politicas educativas en el País Vasco durante el siglo xx, edited by Paulí Dávila, 89-142. Madrid: Biblioteca Nueva, 2004.

Zufía, Pedro. Las escuelas de barriada en Vizcaya. Bilbao: Diputación de Vizcaya, 1930. 